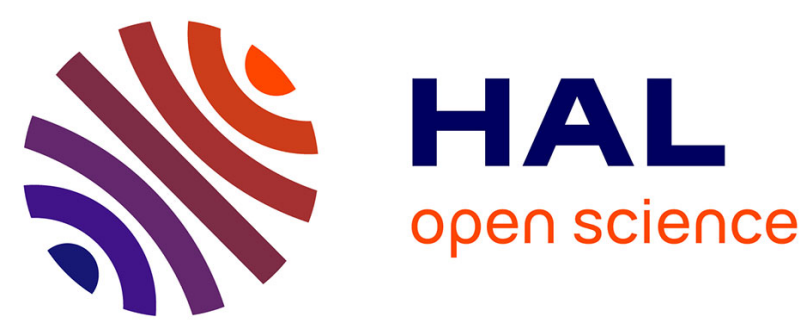

\title{
Equivalence conditions between linear Lagrangian finite element and node-centred finite volume schemes for conservation laws in cylindrical coordinates
}

Dante de Santis, Gianluca Geraci, Alberto Guardone

\section{- To cite this version:}

Dante de Santis, Gianluca Geraci, Alberto Guardone. Equivalence conditions between linear Lagrangian finite element and node-centred finite volume schemes for conservation laws in cylindrical coordinates. International Journal for Numerical Methods in Fluids, 2014, 74 (7), pp.514-542. 10.1002/fld.3862 . hal-00935561

\section{HAL Id: hal-00935561 \\ https://hal.inria.fr/hal-00935561}

Submitted on 23 Jan 2014

HAL is a multi-disciplinary open access archive for the deposit and dissemination of scientific research documents, whether they are published or not. The documents may come from teaching and research institutions in France or abroad, or from public or private research centers.
L'archive ouverte pluridisciplinaire HAL, est destinée au dépôt et à la diffusion de documents scientifiques de niveau recherche, publiés ou non, émanant des établissements d'enseignement et de recherche français ou étrangers, des laboratoires publics ou privés. 


\title{
Equivalence conditions between linear Lagrangian finite element and node-centred finite volume schemes for conservation laws in cylindrical coordinates
}

\author{
D. De Santis ${ }^{1}$, G. Geraci ${ }^{1}$ and A. Guardone $e^{2, *, \dagger}$ \\ ${ }^{1}$ INRIA Bordeaux-Sud-Ouest, Ł̀quipe-projet Bacchus, 200 Avenue de la Vieille Tour, 33405 Talence, France \\ ${ }^{2}$ Department of Aerospace Science and Technology, Politecnico di Milano, Via La Masa 34, 20156 Milano, Italy
}

\section{SUMMARY}

A complete set of equivalence conditions, relating the mass-lumped Bubnov-Galerkin finite element (FE) scheme for Lagrangian linear elements to node-centred finite volume (FV) schemes, is derived for the first time for conservation laws in a three-dimensional cylindrical reference. Equivalence conditions are used to devise a class of FV schemes, in which all grid-dependent quantities are defined in terms of FE integrals. Moreover, all relevant differential operators in the FV framework are consistent with their FE counterparts, thus opening the way to the development of consistent hybrid FV/FE schemes for conservation laws in a three-dimensional cylindrical coordinate system. The two-dimensional schemes for the polar and the axisymmetrical cases are also explicitly derived. Numerical experiments for compressible unsteady flows, including expanding and converging shock problems and the interaction of a converging shock waves with obstacles, are carried out using the new approach. The results agree fairly well with one-dimensional simulations and simplified models. Copyright (C) 2013 John Wiley \& Sons, Ltd.

Received 24 July 2013; Revised 21 October 2013; Accepted 22 October 2013

KEY WORDS: euler equations; cylindrical coordinates; finite element schemes; finite volume schemes

\section{INTRODUCTION}

Starting from the pioneering work of Goldstine and von Neumann [1], Brode [2], Payne [3] and Sod [4], numerous numerical schemes have been proposed for the solution of the compressible flow equations in a cylindrical reference. Relevant applications are, for example, nozzle flows, detonations, astrophysical flows, inertial confinement fusion (ICF), sonoluminescence, explosions and earthquakes [5]. Indeed, in a cylindrical reference system, the symmetries of these physical problems can be easily enforced to reduce the complexity of the discrete problem. The correct representation of secondary flow field features such as shock front instabilities, details of the detonation front in the case of chemically reacting flows (detonation waves) and acoustics requires space-accurate and time-accurate numerical schemes capable of tracking the shock front correctly while at the same time resolving with high accuracy smooth flow regions [6]. For example, shock front instabilities are currently being investigated in connection to ICF, because the occurrence of asymmetric flow patterns may possibly result in the reduction of performance of ICF capsules.

Liu and collaborators [7] applied the high-resolution total variation diminishing (TVD) technique of Harten [8] to spherical explosions and implosions in air under the one-dimensional approximation. Li showed that WENO finite volume (FV) schemes can achieve both high-order accuracy and conservation in cylindrical and spherical geometries [9]. In two-dimensional cylindrical framework,

\footnotetext{
*Correspondence to: A. Guardone, Department of Aerospace Science and Technology, Politecnico di Milano, Via La Masa 34, 20156 Milano, Italy.

†E-mail: alberto.guardone@polimi.it
} 
Dutta and collaborators applied a front tracking algorithm to the simulation of the onset and dynamics of the spherical Richtmyer-Meshkov instability for axisymmetric flows [10]. More recently, Maire proposed a cell-centred Lagrangian formulation to capture axisymmetrical, that is, spherical, imploding shock waves [11]; Illenseer and Duschl generalized the two-dimensional central upwind to curvilinear grids [12]; Clain and collaborators extended a multi-slope MUSCL scheme to cylindrical coordinates [13]. In the framework of Lagrangian or ALE solvers, the explosion and implosion problems in two spatial dimensions were solved by Cheng and Shu using a cell-centred Lagrangian scheme [14]; Loubère and others used the reconnection algorithm for ALE framework in cylindrical coordinates [15].

The present approach moves from the mixed FV/finite element (FE) approach [16], which has been already successfully applied to the multidimensional Cartesian case $[17,18]$ and the cylindrical case with axial symmetry [19], within the throughflow approximation [20] and more recently, in a unified fashion form for orthogonal coordinate systems [21]. To link these two different numerical schemes, it is necessary to introduce suitable equivalence conditions that relate the FV metrics, that is, cell volumes and integrated normals, to the FE integrals. Equivalence conditions in Cartesian coordinate [17, 18] and in cylindrical axisymmetrical form [19] were derived by neglecting high-order contributions from FE schemes. In the present paper, equivalence conditions for the description in a three-dimensional cylindrical coordinates reference system are derived for the first time without introducing any approximation into the FE discrete expression of the divergence operator. A hybrid FE/FV TVD scheme is devised, which simplifies to the first-order FV upwind scheme of Roe close to flow discontinuities and to the second-order FE approximation in smooth flow regions.

The present paper is structured as follows. In Section 2, the FE and FV schemes are briefly described for a scalar conservation law in three spatial dimensions. Equivalence conditions are demonstrated in this case. The scheme is particularized to two-dimensional problems, namely, to the axisymmetric ( $Z-R$ plane) and the polar $(R-\theta)$ representation. The extension to the system of Euler equations for compressible flow is sketched in Section 3. In Section 4, numerical simulations are presented for the explosion and implosion problem in the cylindrical coordinates, including both the axisymmetric and the polar cases, and are compared with one-dimensional simulations. In Section 5, final remarks and comments are given.

\section{FINITE VOLUME/ELEMENT SCHEMES FOR A SCALAR CONSERVATION LAW IN CYLINDRICAL COORDINATES}

In the present section, the FV (Section 2.1) and the FE (Section 2.2) discrete form of the exemplary scalar conservation law

$$
\frac{\partial u}{\partial t}+\nabla^{\varnothing} \cdot f^{\varnothing}=0
$$

in the cylindrical reference shown in Figure 1, with $Z, R$ and $\theta$ axial, radial and angular coordinates, respectively, are derived. In (1), $u \in \mathbb{R}$ and $\boldsymbol{f}^{\varnothing}=\boldsymbol{f}^{\varnothing}(u) \in \mathbb{R}^{3}$ are the scalar unknown and the flux vector with components in $Z, R$ and $\theta$, respectively. The divergence operator in cylindrical coordinates is

$$
\nabla^{\varnothing} \cdot \boldsymbol{f}^{\varnothing}=\frac{\partial f_{Z}}{\partial Z}+\frac{1}{R} \frac{\partial\left(R f_{R}\right)}{\partial R}+\frac{1}{R} \frac{\partial f_{\theta}}{\partial \theta}
$$

where $f_{Z}, f_{R}, f_{\theta} \in \mathbb{R}$ and where $\boldsymbol{f}^{\varnothing}=f_{Z} \hat{\boldsymbol{Z}}+f_{R} \hat{\boldsymbol{R}}+f_{\theta} \hat{\boldsymbol{\theta}}$ and $\hat{\boldsymbol{Z}}, \hat{\boldsymbol{R}}$ and $\hat{\boldsymbol{\theta}}$ are the axial, radial and angular versors, respectively. The singularity at the origin of the reference coordinate system can be removed formally by multiplying by the radial coordinate $R$. Moreover, the modified conservation law already embeds the correct treatment of the origin [22]. Therefore, the model equation is rewritten as

$$
\frac{\partial(R u)}{\partial t}+R \nabla^{\varnothing} \cdot \boldsymbol{f}^{\varnothing}=0 .
$$




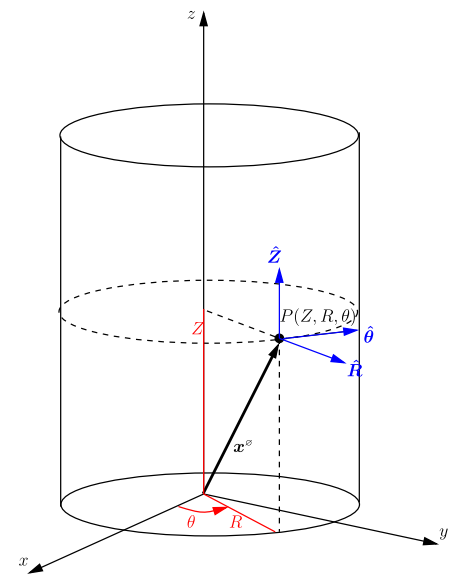

\subsection{Finite volume scheme}

The differential Eq. (2) is now discretized in space by the means of the FV method. The domain $\Omega$ is discretized into $N$ non-overlapping cells; $\Omega=\bigcup_{i=1}^{N} \mathcal{C}_{i}$. Integrating Eq. (2) on a cell $\mathcal{C}_{i}$, one obtains

$$
\int_{\mathcal{C}_{i}} \frac{\partial(R u)}{\partial t} \mathrm{~d} \Omega^{\varnothing}+\oint_{\partial \mathcal{C}_{i}} R \boldsymbol{f}^{\varnothing} \cdot \boldsymbol{n}^{\varnothing} \mathrm{d} \Sigma^{\varnothing}-\int_{\mathcal{C}_{i}} \boldsymbol{f}^{\varnothing} \cdot \hat{\boldsymbol{R}} \mathrm{d} \Omega^{\varnothing}=0
$$

where the divergence theorem has been used. Note that in cylindrical coordinates, the differentials in the volume integrals are defined as $\mathrm{d} \Omega^{\varnothing}=R \mathrm{~d} R \mathrm{~d} Z \mathrm{~d} \theta$, and in an analogous way for the boundary integrals. The normal versor is defined as $\boldsymbol{n}^{\varnothing}=n_{Z} \hat{\boldsymbol{Z}}+n_{R} \hat{\boldsymbol{R}}+n_{\theta} \hat{\boldsymbol{\theta}}$.

In the node-centred approach considered here, each cell surrounds only a node $i \in \mathcal{N}$, where $\mathcal{N}$ is the set of all nodes of the triangulation. On each cell, the cell-average state is defined as

$$
u\left(x^{\varnothing}, t\right) \simeq u_{i}(t)=\frac{1}{V_{i}} \int_{\mathcal{C}_{i}} u\left(x^{\varnothing}, t\right) \mathrm{d} \Omega^{\varnothing},
$$

where $\boldsymbol{x}^{\varnothing}=Z \hat{\boldsymbol{Z}}+R \hat{\boldsymbol{R}}(\theta)$ is the position vector in cylindrical coordinates and $V_{i}$ is the volume of the cell $\mathcal{C}_{i}$, that is, $V_{i}=\int_{\mathcal{C}_{i}} \mathrm{~d} \Omega^{\varnothing}$. Then, Eq. (3) reads

$$
V_{i}^{\varnothing} \frac{\mathrm{d} u_{i}}{\mathrm{~d} t}=-\oint_{\partial \mathcal{C}_{i}} R \boldsymbol{f}^{\varnothing} \cdot \boldsymbol{n}^{\varnothing} \mathrm{d} \Sigma^{\varnothing}+\int_{\mathcal{C}_{i}} \boldsymbol{f}^{\varnothing} \cdot \hat{\boldsymbol{R}} \mathrm{d} \Omega^{\varnothing}
$$

where the following quantity was introduced

$$
V_{i}^{\varnothing} \stackrel{\text { def }}{=} \int_{\mathcal{C}_{i}} R \mathrm{~d} \Omega^{\varnothing}
$$

and for simplicity, the differentials have been omitted in the integrals of Eq. (4).

The boundary integral in Eq. (4) can be split into the contributions of the internal cell interfaces and of the boundary faces as follows:

$$
\oint_{\partial \mathcal{C}_{i}} R \boldsymbol{f}^{\varnothing} \cdot \boldsymbol{n}^{\varnothing} \mathrm{d} \Sigma^{\varnothing}=\sum_{k \in \mathcal{N}_{i, \neq}} \int_{\partial \mathcal{C}_{i k}} R \boldsymbol{f}^{\varnothing} \cdot \boldsymbol{n}_{i}^{\varnothing} \mathrm{d} \Sigma^{\varnothing}+\int_{\partial \mathcal{C}_{i}^{\partial}} R \tilde{\boldsymbol{f}}^{\varnothing} \cdot \boldsymbol{n}^{\varnothing} \mathrm{d} \Sigma^{\varnothing}
$$

where $\tilde{f}^{\varnothing}=f^{\varnothing}(\tilde{u})$ is the flux which takes into account the boundary conditions and $\tilde{u}$ is the boundary state. In the previous equation, $\mathcal{N}_{i, \neq}$ indicates the set of the FVs $\mathcal{C}_{k}$ which share a portion 
of their boundary with $\mathcal{C}_{i}$, except $\mathcal{C}_{i}$ itself. $\partial \mathcal{C}_{i k}=\partial \mathcal{C}_{i} \cap \partial \mathcal{C}_{k} \neq \emptyset, k \neq i$ is the so-called interface of the cell between $\mathcal{C}_{i}$ and $\mathcal{C}_{k}$. The superscript $\partial$ indicates the boundary of the computational domain. It is now possible to rewrite Eq. (4) as follows:

$$
V_{i}^{\varnothing} \frac{\mathrm{d} u_{i}}{\mathrm{~d} t}=-\sum_{k \in \mathcal{N}_{i, \neq}} \int_{\partial \mathcal{C}_{i k}} R \boldsymbol{f}^{\varnothing} \cdot \boldsymbol{n}_{i}^{\varnothing} \mathrm{d} \Sigma^{\varnothing}+\int_{\mathcal{C}_{i}} \boldsymbol{f}^{\varnothing} \cdot \hat{\boldsymbol{R}} \mathrm{d} \Omega^{\varnothing}-\int_{\partial \mathcal{C}_{i}^{\partial}} R \tilde{\boldsymbol{f}}^{\varnothing} \cdot \boldsymbol{n}^{\varnothing} \mathrm{d} \Sigma^{\varnothing} .
$$

Due to the piecewise-constant approximation of the solution on each cell, at the interface $\mathcal{C}_{i k}$, a numerical flux $\boldsymbol{f}_{i k}^{\varnothing}$ must be introduced. As standard practice in FV methods, the numerical flux $\boldsymbol{f}_{i k}^{\varnothing}$, as well as the boundary flux $\tilde{\boldsymbol{f}}_{i}^{\varnothing}$, are assumed constant on the cell interface and the boundary faces, respectively. Therefore, the following approximations are obtained:

$$
\begin{gathered}
\int_{\partial \mathcal{C}_{i k}} R \boldsymbol{f}^{\varnothing} \cdot \boldsymbol{n}_{i}^{\varnothing} \mathrm{d} \Sigma^{\varnothing} \simeq \boldsymbol{f}_{i k}^{\varnothing} \cdot \int_{\partial \mathcal{C}_{i k}} R \boldsymbol{n}_{i}^{\varnothing} \mathrm{d} \Sigma^{\varnothing}=\boldsymbol{f}_{i k}^{\varnothing} \cdot \boldsymbol{v}_{i k}^{\varnothing}, \\
\int_{\partial \mathcal{C}_{i}^{\partial}} R \tilde{\boldsymbol{f}}^{\varnothing} \cdot \boldsymbol{n}_{i}^{\varnothing} \mathrm{d} \Sigma^{\varnothing} \simeq \tilde{\boldsymbol{f}}_{i}^{\varnothing} \cdot \int_{\partial \mathcal{C}_{i}^{\partial}} R \boldsymbol{n}_{i}^{\varnothing} \mathrm{d} \Sigma^{\varnothing}=\tilde{\boldsymbol{f}}_{i}^{\varnothing} \cdot \boldsymbol{v}_{i}^{\partial, \varnothing},
\end{gathered}
$$

where the FV metric quantities has been introduced

$$
\boldsymbol{v}_{i k}^{\varnothing} \stackrel{\text { def }}{=} \int_{\partial \mathcal{C}_{i k}} R \boldsymbol{n}_{i}^{\varnothing} \mathrm{d} \Sigma^{\varnothing} \quad \text { and } \quad \boldsymbol{v}_{i}^{\partial, \varnothing} \stackrel{\text { def }}{=} \int_{\partial \mathcal{C}_{i}^{\partial}} R \boldsymbol{n}_{i}^{\varnothing} \mathrm{d} \Sigma^{\varnothing} .
$$

The final form for the node-centred FV scheme in the node-pair framework reads

$$
V_{i}^{\varnothing} \frac{\mathrm{d} u_{i}}{\mathrm{~d} t}=-\sum_{k \in \mathcal{N}_{i, \neq}} \boldsymbol{f}_{i k}^{\varnothing} \cdot \boldsymbol{v}_{i k}^{\varnothing}+\boldsymbol{f}_{i}^{\varnothing} \cdot \widehat{\boldsymbol{V}}_{i}^{\varnothing}-\tilde{\boldsymbol{f}}_{i}^{\varnothing} \cdot \boldsymbol{v}_{i}^{\partial, \varnothing},
$$

where $\widehat{\boldsymbol{V}}_{i}^{\varnothing} \stackrel{\text { def }}{=} \int_{\mathcal{C}_{i}} \hat{\boldsymbol{R}} \mathrm{d} \Omega^{\varnothing}$.

\subsection{Finite element scheme}

The FE discretization of the scalar conservation law (2) is now obtained. Assuming linear Lagrangian test functions $\varphi_{h} \in V_{h} \subset H^{1}(\Omega)$, the weak formulation of (2) reads [23]

$$
\int_{\Omega_{i}} R \varphi_{i} \frac{\partial u}{\partial t} \mathrm{~d} \Omega^{\varnothing}+\int_{\Omega_{i}} R \varphi_{i} \nabla^{\varnothing} \cdot f^{\varnothing}(u) \mathrm{d} \Omega^{\varnothing}=0, \quad \forall i \in \mathcal{N},
$$

where $\Omega_{i}$ is the compact support of the test function $\varphi_{i}$. By integrating by parts, one obtains

$$
\begin{aligned}
\int_{\Omega_{i}} R \varphi_{i} \frac{\partial u}{\partial t} \mathrm{~d} \Omega^{\varnothing}= & \int_{\Omega_{i}} R \boldsymbol{f}^{\varnothing}(u) \cdot \nabla^{\varnothing} \varphi_{i} \mathrm{~d} \Omega^{\varnothing}+\int_{\Omega_{i}} \varphi_{i} \boldsymbol{f}^{\varnothing}(u) \cdot \nabla^{\varnothing} R \mathrm{~d} \Omega^{\varnothing} \\
& -\int_{\partial \Omega_{i}^{\partial}} R \varphi_{i} \boldsymbol{f}^{\varnothing}(\tilde{u}) \cdot \boldsymbol{n}^{\varnothing} \mathrm{d} \Sigma^{\varnothing}=0 .
\end{aligned}
$$

By approximating the conservative variable using shape functions $\varphi_{h} \in V_{h}, u\left(\boldsymbol{x}^{\varnothing}, t\right) \simeq u_{h}\left(\boldsymbol{x}^{\varnothing}, t\right)=$

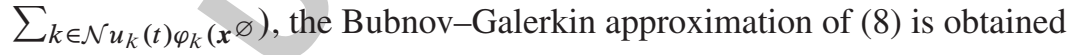

$$
\begin{aligned}
\sum_{k \in \mathcal{N}_{i}} M_{i k}^{\varnothing} \frac{\mathrm{d} u_{k}}{\mathrm{~d} t}= & \int_{\Omega_{i}} R \boldsymbol{f}^{\varnothing}\left(u_{h}\right) \cdot \nabla^{\varnothing} \varphi_{i} \mathrm{~d} \Omega^{\varnothing}+\int_{\Omega_{i}} \varphi_{i} \boldsymbol{f}^{\varnothing}\left(u_{h}\right) \cdot \nabla^{\varnothing} R \mathrm{~d} \Omega^{\varnothing} \\
& -\int_{\partial \Omega_{i}^{\partial}} R \varphi_{i} \boldsymbol{f}^{\varnothing}\left(\tilde{u}_{h}\right) \cdot \boldsymbol{n}^{\varnothing} \mathrm{d} \Sigma^{\varnothing},
\end{aligned}
$$

where $\mathcal{N}_{i}$ is the set of shape functions $\varphi_{k}$ whose support $\Omega_{k}$ shares a portion with $\Omega_{i}$ of $\varphi_{i}$ and where

$$
M_{i k}^{\varnothing} \stackrel{\text { def }}{=} \int_{\Omega_{i k}} R \varphi_{i} \varphi_{k} \mathrm{~d} \Omega^{\varnothing},
$$


with $\Omega_{i k}=\Omega_{i} \cap \Omega_{k}$, is indicated in the following as the $R$-averaged mass matrix. The flux function is expanded with the same basis functions used for the unknown, namely,

$$
\boldsymbol{f}^{\varnothing}\left(u_{h}\right)=\boldsymbol{f}^{\varnothing}\left(\sum_{k \in \mathcal{N}} u_{k}(t) \varphi_{k}\left(\boldsymbol{x}^{\varnothing}\right)\right) \simeq \sum_{k \in \mathcal{N}} \boldsymbol{f}_{k}^{\varnothing} \varphi_{k}\left(\boldsymbol{x}^{\varnothing}\right),
$$

in which $\boldsymbol{f}_{k}^{\varnothing}=\boldsymbol{f}^{\varnothing}\left(u_{k}(t)\right)$. The so-called flux re-interpolation technique above introduces a fourthorder approximation in the evaluation of the integral of the numerical fluxes [24]. By substituting the flux expansion into the Bubnov-Galerkin scheme (10), the final form of the FE scheme reads

$$
\begin{aligned}
\sum_{k \in \mathcal{N}_{i}} M_{i k}^{\varnothing} \frac{\mathrm{d} u_{k}}{\mathrm{~d} t}= & \sum_{k \in \mathcal{N}_{i}} \boldsymbol{f}_{k}^{\varnothing} \cdot \int_{\Omega_{i k}} R \varphi_{k} \nabla^{\varnothing} \varphi_{i} \mathrm{~d} \Omega^{\varnothing}+\sum_{k \in \mathcal{N}_{i}} \boldsymbol{f}_{k}^{\varnothing} \cdot \int_{\Omega_{i k}} \varphi_{i} \varphi_{k} \nabla^{\varnothing} R \mathrm{~d} \Omega^{\varnothing} \\
& -\sum_{k \in \mathcal{N}_{i}^{\partial}} \tilde{\boldsymbol{f}}_{k}^{\varnothing} \cdot \int_{\partial \Omega_{i k}^{\partial}} R \varphi_{i} \varphi_{k} \boldsymbol{n}^{\varnothing} \mathrm{d} \Sigma^{\varnothing},
\end{aligned}
$$

where $\partial \Omega_{i k}^{\partial}=\partial \Omega_{i} \cap \partial \Omega_{k} \cap \partial \Omega$, and $\mathcal{N}^{\partial}$ indicate the set of all the boundary nodes. The notation $\tilde{\boldsymbol{f}}_{k}^{\varnothing}=\boldsymbol{f}^{\varnothing}\left(\tilde{u_{k}}\right)$ has been used to introduce the flux on boundary, which takes into account the imposition of the boundary conditions.

2.2.1. Finite element scheme in node-pair form. As demonstrated in Appendix A, the elementbased representation of the FE discrete problem (11) can be recast into its equivalent node-pair form as follows:

$$
\begin{aligned}
\sum_{k \in \mathcal{N}_{i}} M_{i k}^{\varnothing} \frac{\mathrm{d} u_{k}}{\mathrm{~d} t}= & -\sum_{k \in \mathcal{N}_{i}, \neq}\left(\boldsymbol{f}_{i k}^{\varnothing} \cdot \boldsymbol{\eta}_{i k}^{\varnothing}-\frac{\boldsymbol{f}_{k}^{\varnothing}-\boldsymbol{f}_{k}^{\varnothing}}{2} \cdot \zeta_{i k}^{\varnothing}\right)+\boldsymbol{f}_{i}^{\varnothing} \cdot \widehat{\boldsymbol{L}}_{i}^{\varnothing} \\
& -\sum_{k \in \mathcal{N}_{i, \neq}^{\partial}} \frac{\tilde{\boldsymbol{f}}_{k}^{\varnothing}-\tilde{\boldsymbol{f}}_{i}^{\varnothing}}{2} \cdot \chi_{i k}^{\partial, \varnothing}-\tilde{\boldsymbol{f}}_{i}^{\varnothing} \cdot \xi_{i}^{\partial, \varnothing},
\end{aligned}
$$

where $\mathcal{N}_{i, \neq}=\mathcal{N}_{i} \backslash\{i\}$ and $\mathcal{N}_{i, \neq}^{\partial}=\mathcal{N}_{i}^{\partial} \backslash\{i\}$ and where the following metrics quantities were introduced:

$$
\begin{array}{ccc}
M_{i k}^{\varnothing} \stackrel{\text { def }}{=} \int_{\Omega_{i k}} R \varphi_{i} \varphi_{k} \mathrm{~d} \Omega^{\varnothing}, & \eta_{i k}^{\varnothing} \stackrel{\text { def }}{=} \int_{\Omega_{i k}} R\left(\varphi_{i} \nabla^{\varnothing} \varphi_{k}-\varphi_{k} \nabla^{\varnothing} \varphi_{i}\right) \mathrm{d} \Omega^{\varnothing}, & \zeta_{i k}^{\varnothing} \stackrel{\text { def }}{=} \int_{\Omega_{i k}} \varphi_{i} \varphi_{k} \hat{\boldsymbol{R}} \mathrm{d} \Omega^{\varnothing}, \\
\widehat{\boldsymbol{L}}_{i} \stackrel{\text { def }}{=} \int_{\Omega_{i}} \varphi_{i} \hat{\boldsymbol{R}} \mathrm{d} \Omega^{\varnothing}, & \chi_{i k}^{\partial, \varnothing} \stackrel{\text { def }}{=} \int_{\partial \Omega_{i k}^{\partial}} R \varphi_{i} \varphi_{k} \boldsymbol{n}^{\varnothing} \mathrm{d} \Sigma^{\varnothing} & \boldsymbol{\xi}_{i}^{\partial, \varnothing} \stackrel{\text { def }}{=} \int_{\partial \Omega_{i k}^{\partial}} R \varphi_{i} \boldsymbol{n}^{\varnothing} \mathrm{d} \Sigma^{\varnothing} .
\end{array}
$$

Note that in this case, $\boldsymbol{f}_{i k}^{\varnothing}=\frac{1}{2}\left(\boldsymbol{f}_{i}^{\varnothing}+\boldsymbol{f}_{k}^{\varnothing}\right)$, because a continuous approximation of the solution has been used. Note that this simple central approximation results in an unstable scheme and a suitable streamline stabilization must be added. To this purpose, FV stabilization techniques can be used, thanks to the equivalence conditions demonstrated in the succeeding texts.

By lumping the $R$-averaged mass matrix, namely, by introducing the approximation

$$
\sum_{k \in \mathcal{N}_{i}} M_{i k}^{\varnothing} \frac{\mathrm{d} u_{k}}{\mathrm{~d} t} \simeq L_{i}^{\varnothing} \frac{\mathrm{d} u_{i}}{\mathrm{~d} t}
$$

where $L_{i}^{\varnothing}=\sum_{k \in \mathcal{N}_{i}} M_{i k}^{\varnothing}$, the final node-pair FE discrete counterpart of (2) reads

$$
\begin{aligned}
L_{i}^{\varnothing} \frac{\mathrm{d} u_{i}}{\mathrm{~d} t}= & -\sum_{k \in \mathcal{N}_{i}, \neq}\left(\boldsymbol{f}_{i k}^{\varnothing} \cdot \boldsymbol{\eta}_{i k}^{\varnothing}-\frac{\boldsymbol{f}_{k}^{\varnothing}-\boldsymbol{f}_{i}^{\varnothing}}{2} \cdot \zeta_{i k}^{\varnothing}\right)+\boldsymbol{f}_{i}^{\varnothing} \cdot \widehat{\boldsymbol{L}}_{i}^{\varnothing} \\
& -\sum_{k \in \mathcal{N}_{i, \neq}^{\partial}} \frac{\tilde{\boldsymbol{f}}_{k}^{\varnothing}-\tilde{\boldsymbol{f}}_{i}^{\varnothing}}{2} \cdot \chi_{i k}^{\partial, \varnothing}-\tilde{\boldsymbol{f}}_{i}^{\varnothing} \cdot \xi_{i}^{\partial, \varnothing},
\end{aligned}
$$


to be compared with its FV counterpart (7). It is worth recalling that the lumping step introduces a non-negligible phase error in time with respect to the standard FE approach [24]; however, the development of FE schemes for conservation laws making use of the full mass matrix is still subject of research and lies beyond the scope of the present study [25].

\subsection{Equivalence conditions}

The equivalence conditions that are required to devise a node-centred FV scheme from FE metrics in cylindrical coordinates are now derived. To this purpose, relevant properties of the node-centred FV and linear Lagrangian FE discretizations are briefly recalled.

Considering FV metric quantities first, because $\boldsymbol{n}_{i}^{\varnothing}=-\boldsymbol{n}_{k}^{\varnothing}$ over $\partial \mathcal{C}_{i k}$, then from (6)

$$
\boldsymbol{v}_{i k}^{\varnothing}=-\boldsymbol{v}_{k i}^{\varnothing},
$$

(FVM-a)

which corresponds to the conservation property of the scheme [26]. From the gradient theorem, one also has

$$
\int_{\mathcal{C}_{i}} \nabla^{\varnothing} R=\oint_{\partial \mathcal{C}_{i}} R \boldsymbol{n}_{i}^{\varnothing}
$$

which, from the definition of FV metric quantities (Section 2.1), gives

$$
\widehat{\boldsymbol{V}}_{i}^{\varnothing}=\sum_{k \in \mathcal{N}_{i, \neq}} \boldsymbol{v}_{i k}^{\varnothing}+\boldsymbol{v}_{i}^{\partial, \varnothing}
$$

In Cartesian coordinates, the previously mentioned identity - the 'volume closure' conditionreduces to the well-known property that the integral of the outward normal over a closed domain is identically zero, and it is easily obtained by applying the Gauss theorem to the gradient of a scalar constant. In the cylindrical reference of interest here, the governing equations are multiplied by the radial coordinate $R$ to formally remove the singularity at the origin, and the relevant integral over the boundary is that of the product of $R$ times the outward normal, which via application of the Gauss theorem provides (FVM-b). Hereinafter, identity (FVM-b) will be referred to as the volume closure condition in a cylindrical reference. In this respect, it is remarkable that the vector quantity $\widehat{\boldsymbol{V}}_{i}^{\varnothing}$, namely, the integral of the versor $\hat{\boldsymbol{R}}$ over $\mathcal{C}_{i}$ is in general not parallel to $\hat{\boldsymbol{R}}$ itself, because $\hat{\boldsymbol{R}}$ is a function of $\theta$.

The last identity is obtained by noting that

$$
3 V_{i}^{\varnothing}=\int_{\mathcal{C}_{i}} R \nabla^{\varnothing} \cdot x^{\varnothing} \mathrm{d} \Omega^{\varnothing}=\oint_{\partial \mathcal{C}_{i}} R \boldsymbol{x}^{\varnothing} \cdot \boldsymbol{n}_{i}^{\varnothing} \mathrm{d} \Sigma^{\varnothing}-\int_{\mathcal{C}_{i}} \hat{\boldsymbol{R}} \cdot \boldsymbol{x}^{\varnothing} \mathrm{d} \Omega^{\varnothing}
$$

By approximating the right hand side of the previously mentioned identity using the FV discretization described in Section 2.1 with a central numerical flux, and by recalling (FVM-b), one has

$$
V_{i}^{\varnothing}=\sum_{k \in \mathcal{N}_{i, \neq}} \frac{x_{k}^{\varnothing}-x_{i}^{\varnothing}}{6} \cdot v_{i k}^{\varnothing} .
$$

Property (FVM-c) implies that the volume integral of the divergence of a linear function, that is, the coordinate vector $\boldsymbol{x}^{\varnothing}$, times $R$ is computed exactly by the second-order centred flux. Moreover, it provides a relation between the metric vector $v_{i k}^{\varnothing}$ and the coefficient $V_{i}^{\varnothing}$ multiplying the time derivative in (7).

Moving now to FE metric quantities for linear Lagrangian elements, from its definition the vector $\eta_{i k}^{\varnothing}$ is anti-symmetric, namely,

$$
\boldsymbol{\eta}_{i k}^{\varnothing}=-\boldsymbol{\eta}_{k i}^{\varnothing},
$$


The second identity is obtained by noting that, cf. (A.6), $\sum_{k \in \mathcal{N}_{i}}\left(\eta_{i k}^{\varnothing}-\zeta_{i k}^{\varnothing}\right)+\xi_{i}^{\partial, \varnothing}=\mathbf{0}$, which gives

$$
\widehat{\boldsymbol{L}}_{i}^{\varnothing}=\sum_{k \in \mathcal{N}_{i, \neq}} \eta_{i k}^{\varnothing}+\xi_{i}^{\partial, \varnothing} .
$$

The last relation is obtained from the following identity:

$$
\begin{aligned}
3 L_{i}^{\varnothing}=\int_{\Omega_{i}} 3 R \varphi_{i} \mathrm{~d} \Omega^{\varnothing} & =\int_{\Omega_{i}} R \varphi_{i} \nabla^{\varnothing} \cdot x^{\varnothing} \mathrm{d} \Omega^{\varnothing} \\
& =\oint_{\partial \Omega_{i}^{\partial}} R \varphi_{i} x^{\varnothing} \cdot \boldsymbol{n}_{i}^{\varnothing} \mathrm{d} \Sigma^{\varnothing}-\int_{\Omega_{i}} \varphi_{i} \boldsymbol{x}^{\varnothing} \cdot \hat{\boldsymbol{R}} \mathrm{d} \Omega^{\varnothing}-\int_{\Omega_{i}} R \boldsymbol{x}^{\varnothing} \cdot \nabla^{\varnothing} \varphi_{i} \mathrm{~d} \Omega^{\varnothing} .
\end{aligned}
$$

By substituting the exact expansion $\boldsymbol{x}^{\varnothing}=\sum_{k \in \mathcal{N}_{i}} \boldsymbol{x}_{k}^{\varnothing} \varphi_{k}$, in the right hand side of the previously mentioned identity, one immediately obtains

$$
3 L_{i}^{\varnothing}=\sum_{k \in \mathcal{N}_{i}^{\partial}} \boldsymbol{x}_{k}^{\varnothing} \cdot \int_{\partial \Omega_{i k}^{\partial}} R \varphi_{i} \varphi_{k} \boldsymbol{n}_{i}^{\varnothing} \mathrm{d} \Sigma^{\varnothing}-\sum_{k \in \mathcal{N}_{i}} \boldsymbol{x}_{k}^{\varnothing} \cdot \int_{\Omega_{i k}} \varphi_{i} \varphi_{k} \hat{\boldsymbol{R}} \mathrm{d} \Omega^{\varnothing}-\sum_{k \in \mathcal{N}_{i}} \boldsymbol{x}_{k}^{\varnothing} \cdot \int_{\Omega_{i k}} R \varphi_{k} \nabla^{\varnothing} \varphi_{i} \mathrm{~d} \Omega^{\varnothing},
$$

which, by resorting to the node-pair representation described in Section 2.2.1 and by substituting (FEM-b), can be written as

$$
L_{i}^{\varnothing}=\frac{1}{6} \sum_{k \in \mathcal{N}_{i, \neq}}\left(\boldsymbol{x}_{k}^{\varnothing}-\boldsymbol{x}_{i}^{\varnothing}\right) \cdot\left(\eta_{i k}^{\varnothing}-\zeta_{i k}^{\varnothing}\right)+\frac{1}{6} \sum_{k \in \mathcal{N}_{i, \neq}^{\partial}}\left(x_{k}^{\varnothing}-\boldsymbol{x}_{i}^{\varnothing}\right) \cdot \chi_{i k}^{\partial, \varnothing} .
$$

It is to be noted that (FEM-b) and (FEM-c) stems from the same integral identities (FVM-b) and (FVM-c) are based upon, respectively. This can be realized by recalling that in the variational formulation, the FE test functions $\varphi_{k}$ are such that $\sum_{k} \varphi_{k}\left(\boldsymbol{x}^{\varnothing}\right)=1$ and $\sum_{k} \nabla^{\varnothing} \varphi_{k}\left(\boldsymbol{x}^{\varnothing}\right)=\mathbf{0}$, $\forall x^{\varnothing} \in \Omega$.

Relations (FVM-a), (FVM-b) and (FVM-c) and relations (FEM-a), (FEM-b) and (FEM-c), derived previously for the FV and FE schemes, respectively, are gathered in Table I, together with their continuous counterparts. Inspection of Table I provides a natural way for constructing an FV scheme that is equivalent to a mass-lumped FE scheme for Lagrangian linear elements as follows. By setting

\begin{tabular}{|c|c|c|c|}
\hline & $\mathrm{a}$ & $\mathrm{b}$ & $\mathrm{c}$ \\
\hline Cont. & $-\boldsymbol{n}_{\text {out }}^{\varnothing}$ & $\int_{\mathcal{C}} \nabla^{\varnothing} R \mathrm{~d} \Omega^{\varnothing}=\oint_{\partial \mathcal{C}} R \boldsymbol{n}^{\varnothing} \mathrm{d} \Sigma^{\varnothing}$ & $\int_{\mathcal{C}} R \nabla^{\varnothing} \cdot x^{\varnothing} \mathrm{d} \Omega^{\varnothing}=\oint_{\partial \mathcal{C}} R x^{\varnothing} \cdot n^{\varnothing} \mathrm{d} \Sigma^{\varnothing}$ \\
\hline $\begin{array}{l}\text { Finite volume } \\
\text { method }\end{array}$ & $v_{i k}^{\varnothing}=-v_{k i}^{\varnothing}$ & $\widehat{\boldsymbol{V}}_{i}^{\varnothing}=\sum_{k \in \mathcal{N}_{i, \neq}} \boldsymbol{v}_{i k}^{\varnothing}+\boldsymbol{v}_{i}^{\partial, \varnothing}$ & $V_{i}^{\varnothing}=\frac{1}{6} \sum_{k \in \mathcal{N}_{i, \neq}}\left(x_{k}^{\varnothing}-x_{i}^{\varnothing}\right) \cdot v_{i k}^{\varnothing}$ \\
\hline FEM & $\eta_{i k}^{\varnothing}=-\eta_{k i}^{\varnothing}$ & $\widehat{\boldsymbol{L}}_{i}^{\varnothing}=\sum_{k \in \mathcal{N}_{i, \neq}} \eta_{i k}^{\varnothing}+\xi_{i}^{\partial, \varnothing}$ & $\begin{array}{c}L_{i}^{\varnothing}=\frac{1}{6} \sum_{k \in \mathcal{N}_{i, \neq}}\left(x_{k}^{\varnothing}-x_{i}^{\varnothing}\right) \cdot\left(\eta_{i k}^{\varnothing}-\zeta_{i k}^{\varnothing}\right) \\
+\frac{1}{6} \sum_{k \in \mathcal{N}_{i, \neq}^{\partial}}\left(x_{k}^{\varnothing}-x_{i}^{\varnothing}\right) \cdot \chi_{i k}^{\partial, \varnothing}\end{array}$ \\
\hline
\end{tabular}

$$
\boldsymbol{v}_{i k}^{\varnothing}=\eta_{i k}^{\varnothing} \quad \text { and } \quad \boldsymbol{v}_{i}^{\partial, \varnothing}=\xi_{i}^{\partial, \varnothing},
$$

Table I. Properties a, b and $\mathrm{c}$ in the continuous case, for finite volume method and for lumped FEM metric quantities in three spatial dimensions. 
from properties (FVM-b) and (FEM-b), one immediately obtains

$$
\widehat{\boldsymbol{V}}_{i}^{\varnothing}=\widehat{\boldsymbol{L}}_{i}^{\varnothing}
$$

The node-centred FV scheme that is equivalent to the corresponding FE scheme for Lagrangian linear elements therefore reads

$$
\mathcal{V}_{i}^{\varnothing} \frac{\mathrm{d} u_{i}}{\mathrm{~d} t}=-\sum_{k \in \mathcal{N}_{i, \neq}} \boldsymbol{f}_{i k}^{\varnothing} \cdot \boldsymbol{\eta}_{i k}^{\varnothing}+\boldsymbol{f}_{i}^{\varnothing} \cdot \widehat{\boldsymbol{L}}_{i}^{\varnothing}-\tilde{\boldsymbol{f}}_{i}^{\varnothing} \cdot \xi_{i}^{\partial, \varnothing},
$$

where the quantity $\mathcal{V}_{i}^{\varnothing}$ is computed from either (FVM-c) or (FEM-c) as

$$
\mathcal{V}_{i}^{\varnothing}=\sum_{k \in \mathcal{N}_{i, \neq}} \frac{x_{k}^{\varnothing}-x_{i}^{\varnothing}}{6} \cdot \eta_{i k}^{\varnothing}=L_{i}^{\varnothing}+\sum_{k \in \mathcal{N}_{i, \neq}} \frac{x_{k}^{\varnothing}-x_{i}^{\varnothing}}{6} \cdot \zeta_{i k}^{\varnothing}-\sum_{k \in \mathcal{N}_{i, \neq}^{\partial}} \frac{x_{k}^{\varnothing}-x_{i}^{\varnothing}}{6} \cdot \chi_{i k}^{\partial, \varnothing} \quad \text { (FVE-c) }
$$

The equivalent FV scheme (15) fulfilling the equivalence conditions (FVE-a), (FVE-a) and (FVE-c) computes a discrete value of the integrals in the first column of Table I that is equal to that obtained by the corresponding FE scheme for Lagrangian linear elements. It is remarkable that, differently from the Cartesian case [17,18], in the cylindrical reference, the volume of the $i$-th FV cell is not coincident with the value of the $i$-th element of the FE lumped mass matrix. Moreover, the shape of the FV cells that guarantees equivalence with the FE discretization still remains to be determined. However, the numerical scheme is fully determined without the explicit knowledge of the FV cell shape, thanks to the relation between volumes FVE-c.

\subsection{Reduction to two spatial dimensions}

Differently, from the Cartesian case, the reduction of the three-dimensional problem into twodimensional ones in case of relevant symmetry of the solution is not straightforward, and it is described in details in the present section for the polar and axisymmetric cases.

2.4.1. Polar symmetry $(R-\theta)$. The polar case is considered first, in which the solution is assumed to be independent from the $Z$ variable, that is, $\partial u(Z, R, \theta, t) / \partial Z=0$. In this case, the scalar conservation law (1) reads

$$
\frac{\partial u}{\partial t}+\nabla^{R \theta} \cdot f^{R \theta}=0
$$

where the flux function $\boldsymbol{f}^{R \theta}=f_{R} \hat{\boldsymbol{R}}+f_{\theta} \hat{\boldsymbol{\theta}}$, with $f_{Z}, f_{\theta} \in \mathbb{R}$. The divergence of $\boldsymbol{f}^{R \theta}$ reads

$$
\nabla^{R \theta} \cdot f^{R \theta}=\frac{1}{R} \frac{\partial\left(R f_{R}\right)}{\partial R}+\frac{1}{R} \frac{\partial f_{\theta}}{\partial \theta} .
$$

The FV discrete counterpart of the scalar conservation law (16) is obtained as in the threedimensional case by multiplying (16) by $R$ and by enforcing its integral form over a finite number of volumes $\mathcal{C}_{i}^{R \theta}$ to obtain

$$
\frac{\mathrm{d}}{\mathrm{d} t} \int_{\mathcal{C}_{i}^{R \theta}} R u \mathrm{~d} \Omega^{R \theta}+\int_{\mathcal{C}_{i}^{R \theta}} R \nabla^{R \theta} \cdot f^{R \theta} \mathrm{d} \Omega^{R \theta}=0,
$$

where $\mathrm{d} \Omega^{R \theta}=R \mathrm{~d} R \mathrm{~d} \theta$. By applying the divergence theorem and introducing the following definitions

$$
\begin{aligned}
& V_{i}^{R \theta} \stackrel{\text { def }}{=} \int_{\mathcal{C}_{i}^{R \theta}} R \mathrm{~d} \Omega^{R \theta} \quad \widehat{\boldsymbol{V}}_{i}^{R \theta} \stackrel{\text { def }}{=} \int_{\mathcal{C}_{i}^{R \theta}} \hat{\boldsymbol{R}} \mathrm{d} \Omega^{R \theta} \\
& \boldsymbol{v}_{i k}^{R \theta} \stackrel{\text { def }}{=} \int_{\partial \mathcal{C}_{i k}^{R \theta}} R \boldsymbol{n}_{i}^{R \theta} \mathrm{d} \Sigma^{R \theta} \quad \boldsymbol{v}_{i}^{\partial, R \theta} \stackrel{\text { def }}{=} \int_{\partial \mathcal{C}_{i}^{\partial, R \theta}} R \boldsymbol{n}^{R \theta} \mathrm{d} \Sigma^{R \theta},
\end{aligned}
$$


Table II. Relevant properties of finite volume method and lumped FEM metric quantities in the polar case $(R-\theta)$.

\begin{tabular}{|c|c|c|c|}
\hline \multicolumn{4}{|c|}{ Polar problem $R-\theta$} \\
\hline & $\mathrm{a}$ & $\mathrm{b}$ & $\mathrm{c}$ \\
\hline Cont. & $\boldsymbol{n}_{\mathrm{in}}^{R \theta}=-\boldsymbol{n}_{\mathrm{out}}^{R \theta}$ & $\begin{array}{l}\int_{\mathcal{C}} \nabla^{R \theta} R \Omega^{R \theta} \\
=\oint_{\partial \mathcal{C}} R n^{R \theta} \Sigma^{R \theta}\end{array}$ & $\begin{aligned} \int_{\mathcal{C}} R \nabla^{R \theta} \cdot x^{R \theta} \Omega^{R \theta} \\
\quad=\oint_{\partial \mathcal{C}} R x^{R \theta} \cdot n^{R \theta} \Sigma^{R \theta}-\int_{\mathcal{C}} \hat{\boldsymbol{R}} \cdot x^{R \theta}\end{aligned}$ \\
\hline $\begin{array}{l}\text { Finite volume } \\
\text { method }\end{array}$ & $v_{i k}^{R \theta}=-v_{k i}^{R \theta}$ & $\widehat{\boldsymbol{V}}_{i}^{R \theta}=\sum_{k \in \mathcal{N}_{i, \neq}} \boldsymbol{v}_{i k}^{R \theta}+\boldsymbol{v}_{i}^{\partial, R \theta}$ & $V_{i}^{R \theta}=\frac{1}{4} \sum_{k \in \mathcal{N}_{i, \neq}}\left(\boldsymbol{x}_{k}^{R \theta}-\boldsymbol{x}_{i}^{R \theta}\right) \cdot v_{i k}^{R \theta}$ \\
\hline FEM & $\eta_{i k}^{R \theta}=-\eta_{k i}^{R \theta}$ & $\widehat{\boldsymbol{L}}_{i}^{R \theta}=\sum_{k \in \mathcal{N}_{i, \neq}} \eta_{i k}^{R \theta}+\xi_{i}^{\partial, R \theta}$ & $\begin{array}{c}L_{i}^{R \theta}=\frac{1}{4} \sum_{k \in \mathcal{N}_{i, \neq}}\left(x_{k}^{R \theta}-x_{i}^{R \theta}\right) \cdot\left(\eta_{i k}^{R \theta}-\zeta_{i k}^{R \theta}\right) \\
+\frac{1}{4} \sum_{k}\left(x_{k}^{R \theta}-x_{i}^{R \theta}\right) \cdot \chi_{i k}^{\partial, R \theta}\end{array}$ \\
\hline
\end{tabular}

the FV expression of (16) is obtained as

$$
V_{i}^{R \theta} \frac{\mathrm{d} u_{i}}{\mathrm{~d} t}=-\sum_{k \in \mathcal{N}_{i, \neq}} \boldsymbol{f}_{i k}^{R \theta} \cdot \boldsymbol{v}_{i k}^{R \theta}+\boldsymbol{f}_{i}^{R \theta} \cdot \widehat{\boldsymbol{V}}_{i}^{R \theta}-\tilde{\boldsymbol{f}}_{i}^{R \theta} \cdot \boldsymbol{v}_{i}^{\partial, R \theta} .
$$

Relevant properties relating the FV metric quantities (18) are reported in Table II.

The lumped FE approximation of (16) is derived by multiplying it by $R$ and by linear Lagrangian test functions $\varphi_{i}$ to obtain the following weak form:

$$
\int_{\Omega_{i}^{R \theta}} R \varphi_{i} \frac{\partial u}{\partial t} \mathrm{~d} \Omega^{R \theta}+\int_{\Omega_{i}^{R \theta}} R \nabla^{R \theta} \cdot f^{R \theta} \mathrm{d} \Omega^{R \theta}=0
$$

which, by the same procedure detailed for the three-dimensional case, immediately gives

$$
\begin{aligned}
L_{i}^{R \theta} \frac{\mathrm{d} u_{i}}{\mathrm{~d} t}= & -\sum_{k \in \mathcal{N}_{i}, \neq}\left(\boldsymbol{f}_{i k}^{R \theta} \cdot \boldsymbol{\eta}_{i k}^{R \theta}-\frac{\boldsymbol{f}_{k}^{R \theta}-\boldsymbol{f}_{i}^{R \theta}}{2} \cdot \zeta_{i k}^{R \theta}\right)+\boldsymbol{f}_{i}^{R \theta} \cdot \widehat{\boldsymbol{L}}_{i}^{R \theta} \\
& -\sum_{k \in \mathcal{N}_{i, \neq}^{\partial}} \frac{\tilde{\boldsymbol{f}}_{k}^{R \theta}-\tilde{\boldsymbol{f}}_{i}^{R \theta}}{2} \cdot \chi_{i k}^{\partial, R \theta}-\tilde{\boldsymbol{f}}_{i}^{R \theta} \cdot \boldsymbol{\xi}_{i}^{\partial, R \theta}
\end{aligned}
$$

where the following definitions of the metric quantities hold

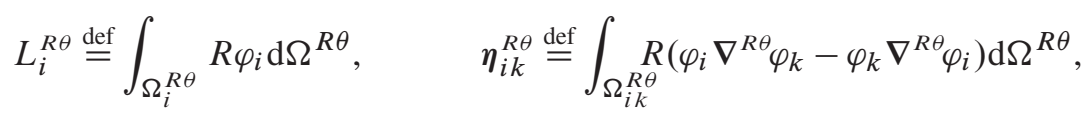

$$
\begin{aligned}
& \zeta_{i k}^{R \theta} \stackrel{\text { def }}{=} \int_{\Omega_{i k}^{R \theta}} \varphi_{i} \varphi_{k} \hat{\boldsymbol{R}} \mathrm{d} \Omega^{R \theta}, \quad \widehat{\boldsymbol{L}}_{i}^{R \theta} \stackrel{\text { def }}{=} \int_{\Omega_{i k}^{R \theta}} \varphi_{i} \hat{\boldsymbol{R}} \mathrm{d} \Omega^{R \theta}, \\
& \chi_{i k}^{\partial, R \theta} \stackrel{\text { def }}{=} \int_{\partial \Omega_{i}^{R \theta}}^{R \varphi_{i}} \varphi_{k} \boldsymbol{n}_{i}^{R \theta} \mathrm{d} \Sigma^{R \theta}, \quad \xi_{i}^{\partial, R \theta} \stackrel{\text { def }}{=} \int_{\partial \Omega_{i k}^{R \theta}}^{R} \varphi_{i} \boldsymbol{n}_{i}^{R \theta} \mathrm{d} \Sigma^{R \theta} .
\end{aligned}
$$

Table III gathers relevant identities relating the previously mentioned FE metric quantities. 
Table III. Relevant properties of finite volume method and lumped FEM metric quantities in the axisymmetric case $(Z-R)$.

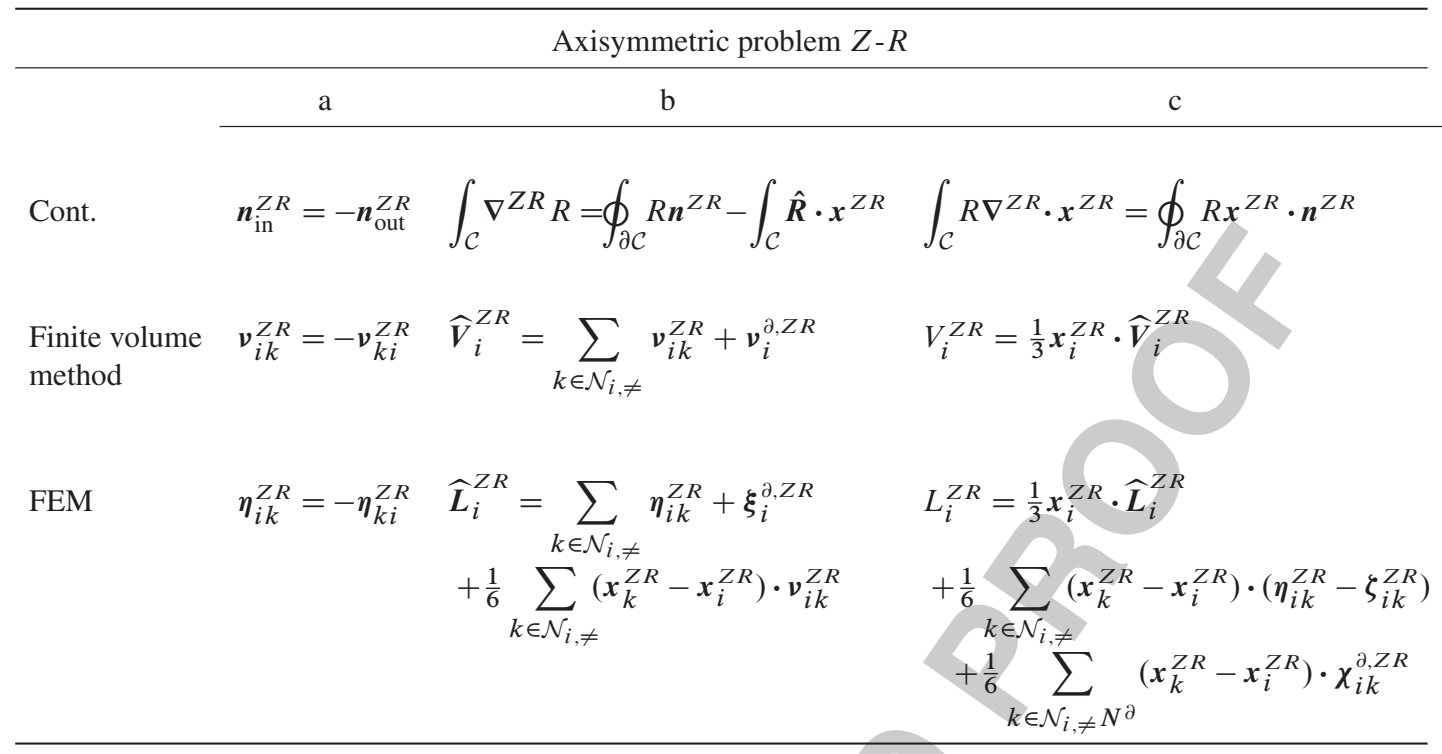

By setting $\boldsymbol{v}_{i k}^{R \theta}=\eta_{i k}^{R \theta}, \boldsymbol{v}_{i}^{\partial, R \theta}=\boldsymbol{\xi}_{i}^{\partial, R \theta}$ and $\widehat{\boldsymbol{V}}_{i}^{R \theta}=\widehat{\boldsymbol{L}}_{i}^{R \theta}$, from the FV and FE identities reported in Table II for the polar case, one obtains the equivalent $\mathrm{FE} / \mathrm{FV}$ scheme as

$$
\mathcal{V}_{i}^{R \theta} \frac{\mathrm{d} u_{i}}{\mathrm{~d} t}=-\sum_{k \in \mathcal{N}_{i, \neq}} \boldsymbol{f}_{i k}^{R \theta} \cdot \boldsymbol{\eta}_{i k}^{R \theta}+\boldsymbol{f}_{i}^{R \theta} \cdot \widehat{\boldsymbol{L}}_{i}^{R \theta}-\tilde{\boldsymbol{f}}_{i}^{R \theta} \cdot \xi_{i}^{\partial, R \theta} .
$$

where the equivalence conditions gives

$$
\mathcal{V}_{i}^{R \theta}=\sum_{k \in \mathcal{N}_{i, \neq}} \frac{x_{k}^{R \theta}-x_{i}^{R \theta}}{4} \cdot \eta_{i k}^{R \theta}=L_{i}^{R \theta}+\sum_{k \in \mathcal{N}_{i, \neq}} \frac{x_{k}^{R \theta}-x_{i}^{R \theta}}{4} \cdot \zeta_{i k}^{R \theta}-\sum_{k \in \mathcal{N}_{i, \neq}^{\partial}} \frac{x_{k}^{R \theta}-x_{i}^{R \theta}}{4} \cdot \chi_{i k}^{\partial, R \theta}
$$

to be confronted with its three-dimensional counterpart (FVE-c).

2.4.2. Axisymmetric case $(Z-R)$. Considering now the axisymmetric case, a symmetry exists with respect to the $\theta$ coordinate, namely, $\partial u(Z, R, \theta, t) / \partial \theta=0$ and the scalar conservation law (1) simplifies to

$$
\frac{\partial u}{\partial t}+\nabla^{Z} R \cdot f^{Z R}+\frac{1}{R} f_{R}=0
$$

where $\boldsymbol{f}^{Z R}=f_{Z} \hat{\mathbf{Z}}+f_{R} \hat{\boldsymbol{R}}$, with $f_{Z}, f_{R} \in \mathbb{R}$ and where the divergence operators are defined as

$$
\nabla^{Z R} \cdot f^{Z R}=\frac{\partial f_{Z}}{\partial Z}+\frac{1}{R} \frac{\partial\left(R f_{R}\right)}{\partial R} .
$$

The geometrical source term $f_{R} / R$ in (23) has no counterpart in the three-dimensional, cf. Eq. (1), and polar, cf. Eq. (16), cases. Although differential operators in the axisymmetric coordinate system are very well known, it is worth recalling here that the definition (24) of the divergence operator is not arbitrary, but it is instead dictated by the peculiar metric of the axisymmetric reference. No alternatives are available if differential identities such as, for example, the Gauss theorem are to be applied in a straightforward manner to the divergence term.

The scalar conservation law (23) is multiplied by the radial coordinate $R$, and its integral form is enforced over a finite number of volumes $\mathcal{C}_{i}^{Z R}$ to obtain

$$
\frac{\mathrm{d}}{\mathrm{d} t} \int_{\mathcal{C}_{i}^{Z R}} R u \mathrm{~d} \Omega^{Z R}+\int_{\mathcal{C}_{i}^{Z R}} R \nabla^{Z R} \cdot f^{Z R} \mathrm{~d} \Omega^{Z R}+\int_{\mathcal{C}_{i}^{Z R}} f_{R} \mathrm{~d} \Omega^{Z R}=0,
$$


with $\mathrm{d} \Omega^{Z R}=\mathrm{d} R \mathrm{~d} Z$. By substituting the following identity in the integral expression previously mentioned,

$$
\int_{\mathcal{C}_{i}^{Z R}} R \nabla^{Z R} \cdot f^{Z R} \mathrm{~d} \Omega^{Z R}=\int_{\mathcal{C}_{i}^{Z R}} \nabla^{Z R} \cdot R f^{Z R} \mathrm{~d} \Omega^{Z R}-\int_{\mathcal{C}_{i}^{Z R}} f^{Z R} \cdot \nabla^{Z R} R \mathrm{~d} \Omega^{Z R}
$$

one immediately obtains

$$
\frac{\mathrm{d}}{\mathrm{d} t} \int_{\mathcal{C}_{i}^{Z R}} R u \mathrm{~d} \Omega^{Z R}=-\oint_{\partial \mathcal{C}_{i}^{Z R}} R \boldsymbol{f}^{Z R} \cdot \boldsymbol{n}_{i}^{Z R} \mathrm{~d} \Sigma^{Z R}
$$

The FV discrete counterpart of (23) is finally obtained as

$$
V_{i}^{Z R} \frac{\mathrm{d} u_{i}}{\mathrm{~d} t}=-\sum_{k \in \mathcal{N}_{i, \neq}} \boldsymbol{f}_{i k}^{Z R} \cdot \boldsymbol{v}_{i k}^{Z R}-\tilde{\boldsymbol{f}}_{i}^{Z R} \cdot \boldsymbol{v}_{i}^{\partial, Z R}
$$

where the following metric quantities have been introduced (Table III)

$$
V_{i}^{Z R} \stackrel{\text { def }}{=} \int_{\mathcal{C}_{i}^{Z R}} R \mathrm{~d} \Omega^{Z R}, \quad \boldsymbol{v}_{i k}^{Z R} \stackrel{\text { def }}{=} \int_{\partial \mathcal{C}_{i k}^{Z R}} R \boldsymbol{n}_{i}^{Z R} \mathrm{~d} \Omega^{Z R} \quad \text { and } \quad \boldsymbol{v}_{i}^{\partial, Z R} \stackrel{\text { def }}{=} \int_{\partial C_{i}^{\partial, Z R}} R \boldsymbol{n}^{Z R} \mathrm{~d} \Sigma^{Z R}
$$

The FE discrete form of (23) is obtained by the following weak form:

$$
\int_{\Omega_{i}^{Z R}} R \varphi_{i} \frac{\partial u}{\partial t} \mathrm{~d} \Omega^{Z R}+\int_{\Omega_{i}^{Z R}} R \varphi_{i} \nabla^{Z R} \cdot f^{Z R}(u) \mathrm{d} \Omega^{Z R}+\int_{\Omega_{i}^{Z R}} \varphi_{i} f_{R} \mathrm{~d} \Omega^{Z R}=0,
$$

which can be recast in the following node-pair form

$$
\begin{aligned}
L_{i}^{Z R} \frac{\mathrm{d} u_{i}}{\mathrm{~d} t}= & -\sum_{k \in \mathcal{N}_{i}, \neq}\left(\boldsymbol{f}_{i k}^{Z R}+\boldsymbol{f}_{i}^{Z R} \cdot \eta_{i k}^{Z R}+\frac{\boldsymbol{f}_{k}^{Z R}-\boldsymbol{f}_{i}^{Z R}}{2} \cdot \zeta_{i k}^{Z R}\right) \\
& -\sum_{k \in \mathcal{N}_{i, \neq}^{\partial}} \frac{\tilde{\boldsymbol{f}}_{k}^{Z R}-\tilde{\boldsymbol{f}}_{i}^{Z R}}{2} \cdot \chi_{i k}^{\partial, Z R}-\tilde{\boldsymbol{f}}_{i}^{Z R} \cdot \xi_{i}^{\partial, Z R},
\end{aligned}
$$

where the FE metric quantities for the axisymmetric case read

$$
\begin{aligned}
\eta_{i k}^{Z R} \stackrel{\text { def }}{=} \int_{\Omega_{i k}^{Z R}} R\left(\varphi_{i} \nabla^{Z R} \varphi_{k}-\varphi_{k} \nabla^{Z R} \varphi_{i}\right) \mathrm{d} \Omega^{Z R}, & \\
L_{i}^{Z R} \stackrel{\text { def }}{=} \sum_{k \in \mathcal{N}_{i}} \int_{\Omega_{i k}^{Z R}} R \varphi_{i} \varphi_{k} \mathrm{~d} \Omega^{Z R}, & \zeta_{i k}^{Z R} \stackrel{\text { def }}{=} \hat{\boldsymbol{R}} \int_{\Omega_{i k}^{Z R}} \varphi_{i} \varphi_{k} \mathrm{~d} \Omega^{Z R}, \\
\chi_{i k}^{\partial, Z R} \stackrel{\text { def }}{=} \int_{\partial \Omega_{i}^{Z R}}^{R \varphi_{i}} \varphi_{k} \boldsymbol{n}_{i}^{Z R} \mathrm{~d} \Sigma^{Z R}, & \xi_{i}^{\partial, Z R} \stackrel{\text { def }}{=} \int_{\partial \Omega_{i k}^{Z R}}^{R \varphi_{i}} \boldsymbol{n}_{i}^{Z R} \mathrm{~d} \Sigma^{Z R} .
\end{aligned}
$$

Relevant relations among the previously mentioned FE metric quantities are gathered in Table III. Note that differently from the three-dimensional case discussed in the previous section, in the definition of $\zeta_{i k}^{Z R}$, the versor $\hat{\boldsymbol{R}}$ is always normal to the versor $\hat{\boldsymbol{Z}}$, and it can be factorized, similarly to the Cartesian two-dimensional.

The equivalent FV/FE is finally obtained by choosing $\boldsymbol{v}_{i k}^{Z R}=\eta_{i k}^{Z R}, \boldsymbol{v}_{i}^{\partial, Z R}=\xi_{i}^{\partial, Z R}, \widehat{\boldsymbol{V}}_{i}^{Z R}=\widehat{\boldsymbol{L}}_{i}^{Z R}$ as follows:

$$
\mathcal{V}_{i}^{Z R} \frac{\mathrm{d} u}{\mathrm{~d} t}=-\sum_{k \in \mathcal{N}_{i}, \neq} \frac{\boldsymbol{f}_{k}^{Z R}+\boldsymbol{f}_{i}^{Z R}}{2} \cdot \boldsymbol{\eta}_{i k}^{Z R}-\tilde{\boldsymbol{f}}_{i}^{Z R} \cdot \xi_{i}^{\partial, Z R},
$$

where from the FE/FV equivalence conditions for the axisymmetric case (Table III), one has

$$
\mathcal{V}_{i}^{Z R}=\sum_{k \in \mathcal{N}_{i, \neq}} \frac{x_{k}^{Z R}-x_{i}^{Z R}}{6} \cdot \eta_{i k}^{Z R}=L_{i}^{Z R}+\sum_{k \in \mathcal{N}_{i, \neq}} \frac{x_{k}^{Z R}-x_{i}^{Z R}}{6} \cdot \zeta_{i k}^{Z R}-\sum_{k \in \mathcal{N}_{i, \neq}^{\partial}} \frac{x_{k}^{Z R}-x_{i}^{Z R}}{6} \cdot \chi_{i k}^{\partial, Z R} .
$$


It is remarkable that differently from the Cartesian case, in which the reduction to two or one spatial problems from the three-dimensional case is straightforward, in the cylindrical reference, such a simplification is to be carried out with care and possibly leads to different results depending on the type of symmetry that is enforced. This is the case for the polar and axisymmetric cases considered here, in which in the former, the additional term $\mathbf{f}_{i}^{R \theta} \cdot \widehat{L}_{i}^{R \theta}$, that has no counterpart in the axisymmetric case, has to be considered.

\section{HYBRID FINITE VOLUME/ELEMENT SCHEMES FOR THE EULER EQUATIONS}

In the present section, the full mixed FV/FE discrete form for the Euler equations is presented including the discretization of the source term and the time derivative. The Euler equations for compressible flows in the cylindrical reference are

$$
\frac{\partial \mathbf{u}^{\varnothing}}{\partial t}+\nabla^{\varnothing} \cdot \mathbf{f}^{\varnothing}=\frac{1}{R} \mathbf{s}^{\varnothing},
$$

where $\mathbf{u}^{\varnothing}(Z, R, \theta, t)=\left(\rho, \boldsymbol{m}^{\varnothing}, E^{t}\right)^{\mathrm{T}}$, with $\boldsymbol{m}^{\varnothing}=\left(m_{Z}, m_{R}, m_{\theta}\right)^{\mathrm{T}}$, is the vector of the conserved variables density, momentum and total energy per unit volume and where the vector of the fluxes $\mathbf{f}^{\varnothing}\left(\mathbf{u}^{\varnothing}\right)$ and of the geometric source terms $\mathbf{s}^{\varnothing}\left(\mathbf{u}^{\varnothing}\right)$ are

$$
\mathbf{f}^{\varnothing}\left(\mathbf{u}^{\varnothing}\right)=\left(\begin{array}{ccc}
m_{Z} & m_{R} & m_{\theta} \\
\frac{m_{Z}^{2}}{\rho}+\Pi & \frac{m_{R} m_{Z}}{\rho} & \frac{m_{\theta} m_{Z}}{\rho} \\
\frac{m_{Z} m_{R}}{\rho} & \frac{m_{R}^{2}}{\rho}+\Pi & \frac{m_{\theta} m_{r}}{\rho} \\
\frac{m_{Z} m_{\theta}}{\rho} & \frac{m_{R} m_{\theta}}{\rho} & \frac{m_{\theta}^{2}}{\rho}+\Pi \\
\frac{m_{Z}}{\rho}\left(E^{t}+\Pi\right) & \frac{m_{R}}{\rho}\left(E^{t}+\Pi\right) & \frac{m_{\theta}}{\rho}\left(E^{t}+\Pi\right)
\end{array}\right), \quad \mathbf{s}^{\varnothing}\left(\mathbf{u}^{\varnothing}\right)=\left(\begin{array}{c}
0 \\
0 \\
\frac{m_{\theta}^{2}}{\rho}+\Pi \\
\frac{m_{\theta} m_{R}}{\rho} \\
0,
\end{array}\right)
$$

where $\Pi=\Pi\left(\mathbf{u}^{\varnothing}\right)$ is the pressure. Under the FV approach, the average value of the source term over the $i$-th cell is approximated as $\mathbf{s}^{\varnothing}\left(\mathbf{u}^{\varnothing}, t\right) \sim \mathrm{s}_{i}^{\varnothing}(t)=\mathbf{s}^{\varnothing}\left(\mathrm{u}_{i}^{\varnothing}(t)\right)$, which provides a first-order spatial approximation of the source function. Therefore,

$$
\int_{\mathcal{C}_{i}} \mathrm{~s}^{\varnothing} \mathrm{d} \Omega^{\varnothing} \simeq \mathrm{s}_{i}^{\varnothing} V_{i}
$$

and the FV approximation of (31) reads

$$
V_{i}^{\varnothing} \frac{\mathrm{d} \mathbf{u}_{i}^{\varnothing}}{\mathrm{d} t}=-\sum_{k \in \mathcal{N}_{i}, \neq} \mathbf{f}_{i k}^{\varnothing} \cdot \boldsymbol{v}_{i k}^{\varnothing}+\mathbf{f}_{i}^{\varnothing} \cdot \widehat{\boldsymbol{V}}_{i}^{\varnothing}+\mathrm{s}_{i}^{\varnothing} V_{i}-\tilde{\mathbf{f}}_{i}^{\varnothing} \cdot \boldsymbol{v}_{i}^{\partial, \varnothing}
$$

with $V_{i}=\int_{\mathcal{C}_{i}} \mathrm{~d} \Omega^{\varnothing}$ and the metric quantities have been defined (Section 2.1). The quantity $\tilde{\mathbf{f}}_{i}^{\varnothing}=\mathbf{f}^{\varnothing}\left(\tilde{\mathbf{u}}_{i}^{\varnothing}\right)$ is the Euler flux evaluated at the state $\tilde{\mathbf{u}}_{i}^{\varnothing}$, which take into account of the boundary conditions.

Considering now the FE approach, the source term is first interpolated $\mathbf{s}^{\varnothing}\left(\mathbf{u}^{\varnothing}\right) \simeq$ $\sum_{k \in \mathcal{N}_{i}} \mathbf{s}_{k}^{\varnothing}(t) \varphi_{k}\left(\boldsymbol{x}^{\varnothing}\right)$ where $\mathbf{s}_{k}^{\varnothing}(t) \stackrel{\text { def }}{=} \mathbf{s}^{\varnothing}\left(\mathbf{u}_{k}^{\varnothing}(t)\right)$, so that

$$
\int_{\Omega_{i}} \varphi_{i} \mathbf{s}^{\varnothing}\left(\mathrm{u}^{\varnothing}\right) \mathrm{d} \Omega^{\varnothing} \simeq \sum_{k \in \mathcal{N}_{i}} \mathbf{s}_{k}^{\varnothing}(t) \int_{\Omega_{i k}} \varphi_{i} \varphi_{k} \mathrm{~d} \Omega^{\varnothing}=\sum_{k \in \mathcal{N}_{i}} M_{i k} \mathbf{s}_{k}^{\varnothing}(t),
$$


where the lumped mass matrix approximation was used, $M_{i k}=\int_{\Omega_{i}} \varphi_{i} \varphi_{k}$. By applying the node-pair FE formulation in Section 2.2 to the complete system of Euler equations, one obtains

$$
\begin{aligned}
L_{i}^{\varnothing} \frac{\mathrm{d} \mathbf{u}_{i}^{\varnothing}}{\mathrm{d} t}= & -\sum_{k \in \mathcal{N}_{i}, \neq}\left(\mathbf{f}_{i k}^{\varnothing} \cdot \eta_{i k}^{\varnothing}-\frac{\mathbf{f}_{k}^{\varnothing}-\mathbf{f}_{i}^{\varnothing}}{2} \cdot \zeta_{i k}^{\varnothing}\right)+\mathbf{f}_{i}^{\varnothing} \cdot \widehat{\boldsymbol{L}}_{i}^{\varnothing}+\mathbf{s}_{i}^{\varnothing} L_{i} \\
& -\sum_{k \in \mathcal{N}_{i, \neq}^{\partial}} \frac{\mathbf{f}_{k}^{\varnothing}-\mathbf{f}_{i}^{\varnothing}}{2} \cdot \chi_{i k}^{\partial, \varnothing}-\tilde{\mathbf{f}}_{i}^{\varnothing} \cdot \xi_{i}^{\partial, \varnothing},
\end{aligned}
$$

where all the metric quantities have already been defined (Section 2.2.1), and the mass lumping approximation $L_{i}=\sum_{k} M_{i k}$ is employed.

By imposing the equivalence conditions (FVE-a), (FVE-a) and (FVE-c), the mixed FE/FV formulation for the Euler system is finally obtained as

$$
\mathcal{V}_{i}^{\varnothing} \frac{\mathrm{d} \mathbf{u}_{i}^{\varnothing}}{\mathrm{d} t}=\mathrm{R}_{i}^{\varnothing}(\mathrm{u}), \quad \text { with } \quad \mathrm{R}_{i}^{\varnothing}(\mathrm{u})=-\sum_{k \in \mathcal{N}_{i}, \neq} \mathbf{f}_{i k}^{\varnothing} \cdot \eta_{i k}^{\varnothing}+\mathbf{f}_{i}^{\varnothing} \cdot \widehat{\boldsymbol{L}}_{i}^{\varnothing}+\mathbf{s}_{i}^{\varnothing} L_{i}-\tilde{\mathbf{f}}_{i}^{\varnothing} \cdot \xi_{i}^{\partial, \varnothing} .
$$

In the following, the reduction of the proposed three-dimensional scheme to polar $(R-\theta)$ and axisymmetric $(Z-R)$ two-dimensional compressible flow is briefly outlined. As discussed in Section 2.4, the polar case is the description of the three-dimensional flow in the $R-\theta$ plane, which is valid if a symmetry with respect to the $Z$ variable exists. In this case, the Euler equations simplify to

$$
\frac{\partial \mathbf{u}^{R \theta}}{\partial t}+\nabla^{R \theta} \cdot \mathbf{f}^{R \theta}=\frac{1}{R} \mathbf{s}^{R \theta}
$$

where $\mathrm{u}^{R \theta}(R, \theta, t)=\left(\rho, \boldsymbol{m}^{R \theta}, E^{t}\right)^{\mathrm{T}}$ is the vector of conservative variables, with $\boldsymbol{m}^{R \theta}=$ $\left(m_{R}, m_{\theta}\right)^{\mathrm{T}}$ momentum vector. The vector of the fluxes $\mathbf{f}^{R \theta}=\mathbf{f}_{R} \hat{\boldsymbol{R}}+\mathbf{f}_{\theta} \hat{\boldsymbol{\theta}}$ and the geometrical source vector $\mathbf{s}^{R \theta}$ are

$$
\mathbf{f}^{R \theta}\left(\mathbf{u}^{R \theta}\right)=\left(\begin{array}{cc}
m_{R} & m_{\theta} \\
\frac{m_{R}^{2}}{\rho}+\Pi & \frac{m_{\theta} m_{r}}{\rho} \\
\frac{m_{R} m_{\theta}}{\rho} & \frac{m_{\theta}^{2}}{\rho}+\Pi \\
\frac{m_{R}}{\rho}\left(E^{t}+\Pi\right) & \frac{m_{\theta}}{\rho}\left(E^{t}+\Pi\right)
\end{array}\right), \quad \mathbf{s}^{R \theta}=\left(\begin{array}{c}
0 \\
\frac{m_{\theta}^{2}}{\rho}+\Pi \\
\frac{m_{\theta} m_{R}}{\rho} \\
0
\end{array}\right),
$$

The equivalent $\mathrm{FV}$ scheme therefore reads

$V_{i}^{R \theta} \frac{\mathrm{d} \mathbf{u}_{i}^{R \theta}}{\mathrm{d} t}=\mathrm{R}_{i}^{R \theta}(\mathrm{u}), \quad$ with $\quad \mathrm{R}_{i}^{R \theta}(\mathrm{u})=-\sum_{k \in \mathcal{N}_{i, \neq}} \mathbf{f}_{i k}^{R \theta} \cdot \eta_{i k}^{R \theta}+\mathbf{f}_{i}^{R \theta} \cdot \widehat{\boldsymbol{L}}_{i}^{R \theta}+\mathrm{s}_{i}^{R \theta} L_{i}^{R \theta}-\tilde{\mathbf{f}}_{i}^{R \theta} \cdot \xi_{i}^{\partial, R \theta}$,

where the metric quantities are defined in Section 2.4.

Considering now the axisymmetrical case, a symmetry exists with respect to the $\theta$ coordinate, namely, $\partial / \partial \theta=0$. Moreover, if the tangential component $m_{\theta}$ of the momentum vector is zero everywhere (no swirl motion), then the Euler equation for a compressible fluid is

$$
\frac{\partial \mathbf{u}^{Z R}}{\partial t}+\nabla^{Z R} \cdot \mathbf{f}^{Z R}+\frac{\hat{\boldsymbol{R}}}{R} \cdot \mathbf{f}^{Z R}=\frac{1}{R} \mathbf{s}^{Z R},
$$


in which the vector of conservative variable is $\mathrm{u}^{Z R}(Z, R, t)=\left(\rho, \boldsymbol{m}^{Z R}, E^{t}\right)^{\mathrm{T}}$ with the $\boldsymbol{m}^{Z R}=$ $\left(m_{Z}, m_{R}\right)^{\mathrm{T}}$ momentum vector. The matrix of the fluxes and the source vector is

$$
\mathbf{f}^{Z R}\left(\mathbf{u}^{Z R}\right)=\left(\begin{array}{cc}
m_{Z} & m_{R} \\
\frac{m_{Z}^{2}}{\rho}+\Pi & \frac{m_{R} m_{Z}}{\rho} \\
\frac{m_{Z} m_{R}}{\rho} & \frac{m_{R}^{2}}{\rho}+\Pi \\
\frac{m_{Z}}{\rho}\left(E^{t}+\Pi\right) & \frac{m_{R}}{\rho}\left(E^{t}+\Pi\right)
\end{array}\right), \quad \mathbf{s}^{Z R}\left(\mathbf{u}^{Z R}\right)=\left(\begin{array}{l}
0 \\
0 \\
\Pi \\
0 .
\end{array}\right)
$$

By applying the procedure in Section 2.4, the equivalent FV scheme is obtained as

$$
\mathcal{V}_{i}^{Z R} \frac{\mathrm{d} \mathbf{u}_{i}^{Z R}}{\mathrm{~d} t}=\mathrm{R}_{i}^{Z R}(\mathrm{u}), \quad \text { with } \quad \mathrm{R}_{i}^{Z R}(\mathrm{u})=-\sum_{k \in \mathcal{N}_{i}, \neq} \mathbf{f}_{i k}^{Z R} \cdot \eta_{i k}^{Z R}+\mathrm{s}_{i}^{Z R} L_{i}^{Z R}-\tilde{\mathbf{f}}_{i}^{Z R} \cdot \xi_{i}^{\partial, Z R},
$$

where the metric quantities are defined in Section 2.4. The construction of an equivalent FV scheme for the Euler equation for one-dimensional problems endowed with cylindrical symmetry is discussed in [21].

In the numerical simulation, the second-order TVD numerical flux has been used

$$
\mathbf{f}_{i k}=\frac{1}{2}\left[\left(\mathbf{f}_{i}+\mathbf{f}_{k}\right) \cdot \boldsymbol{\eta}_{i k}+\left(\Psi_{i k}-\mathbb{I}\right)\left|\hat{\mathrm{A}}_{i k}^{\eta_{i k}}\right|\left(\mathrm{u}_{k}-\mathrm{u}_{i}\right),\right]
$$

with $\hat{\mathrm{A}}_{i k}^{\eta_{i k}}$ the Roe matrix [27], $\Psi_{i k}$ is the limiter diagonal matrix, and $\mathbb{I}$ is the identity matrix. The previously mentioned relation reduce to the first-order upwind scheme for $\Psi_{i k}=0$, whereas for $\Psi_{i k}=\mathbb{I}$, the second-order centred scheme is recovered. The Roe matrix has been evaluated using the approach described in [28], and the van Leer limiter has been used [29]. Note that in the numerical flux has been written for a generic reference system, for this reason; the superscripts were dropped.

The boundary conditions are enforced in a weak way. In particular, the slip wall boundary conditions, which are the only boundary condition used in this work, are imposed by the means of the following flux

$$
\tilde{f}=f\left(\tilde{u}_{\hat{n}}\right), \quad \text { with } \quad \tilde{u}_{\hat{n}}=\left.u\right|_{v_{\perp}=0},
$$

namely the boundary state takes into account that the normal component of the velocity to the wall, $v_{\perp}=\boldsymbol{v} \cdot \hat{\boldsymbol{n}}$, is zero, whit $\hat{\boldsymbol{n}}$ the normal versor to the wall.

The fully discrete form of the Euler equations is now obtained by applying a standard implicit $p$-step backward differentiation formula (BDF) to the time derivative term in (35). An implicit time integration scheme is preferred over explicit ones, such as Runge-Kutta schemes with operator splitting, to prevent numerical errors arising from the unbalancing of the advection and source terms in steady-state computations or in uniform flow regions [26]. Dropping the symbol $\varnothing$ for brevity, the fully discrete form of (31) reads

$$
\frac{\mathcal{V}_{i}}{\Delta t^{n}} \sum_{l=-1}^{p} a_{-l} \mathrm{u}_{i}^{n-l}=\mathrm{R}_{i}^{n+1},
$$

where $p$ is the number of backward steps ( $p+1$ is the order of the BDF scheme), $a_{-l}$ are the coefficients of the BDF scheme, the superscript $n$ indicates discrete time levels and $\Delta t^{n}=t^{n+1}-t^{n}$ is the time step. In the second-order BDF scheme used in the computations, the coefficients $a_{-l}$ read

$$
a_{-1}=\frac{\beta^{2}}{1+\beta}, \quad a_{0}=-(1+\beta), \quad a_{1}=\frac{1+2 \beta}{1+\beta}, \quad \text { with } \quad \beta=\frac{\Delta t^{n}}{\Delta t^{n-1}} .
$$

To preserve second-order accuracy, the computation of the first time step is carried out using a thirdorder Runge-Kutta explicit scheme. The solution of the implicit non-linear system (40) is obtained 
by means of a modified Newton-Raphson scheme, in which a dual time step integration technique is implemented [30]. If a backward Euler discretization for the time derivative in the dual time $\tau$ is considered, for each time step $\Delta \tau$, the following system of linear equation,

$$
\left[\mathcal{V}_{i}\left(\frac{a_{1}}{\Delta t}+\frac{1}{\Delta \tau}\right)-\frac{\partial \mathrm{R}_{i}(\tilde{\mathrm{u}})}{\partial \tilde{\mathrm{u}}}\right] \Delta \tilde{\mathrm{u}}=\mathrm{R}_{i}(\tilde{\mathrm{u}})-\frac{\mathcal{V}_{i}}{\Delta t}\left[a_{1} \tilde{\mathrm{u}}+\sum_{l=0}^{p} a_{-l} \mathrm{u}_{i}^{n-l}\right],
$$

where $\Delta \tilde{\mathrm{u}}=\mathrm{u}^{n+1}-\tilde{\mathrm{u}}$ is solved by means of the SPARSEKIT_Software [31]. Dual time stepping integration is carried starting from the initial value $\tilde{u}=u^{n}$ to reduce the $L_{2}$ norm of the residual to less than $10^{-7}$ times its initial value at $t^{n}$.

The resulting numerical scheme is formally second-order accurate both in space and time for smooth solution, as it has been shown in the two-dimensional axisymmetric formulation [19] or the one-dimensional spherical and cylindrical one [21]. In the presence of shock waves, the scheme is first-order accurate in space.

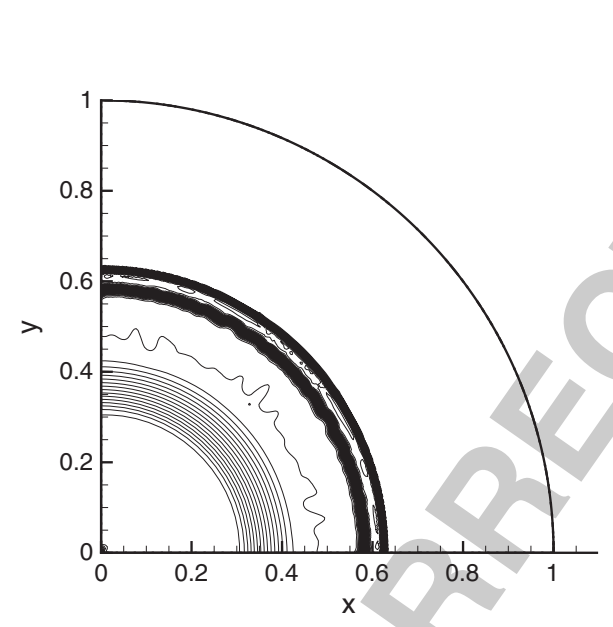

(a)

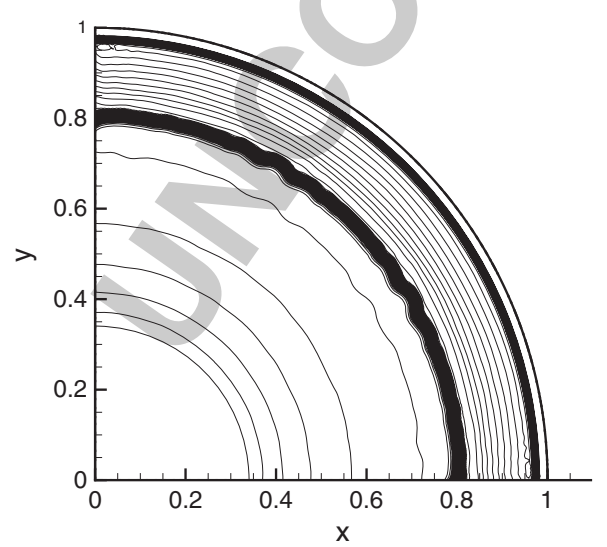

(c)

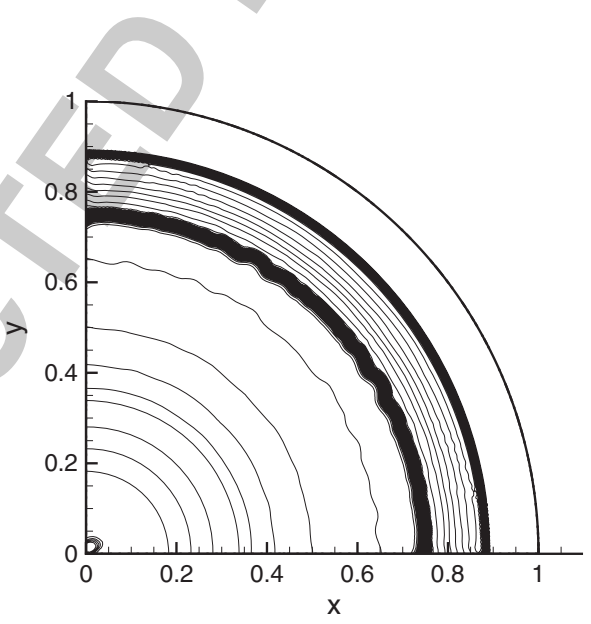

(b)

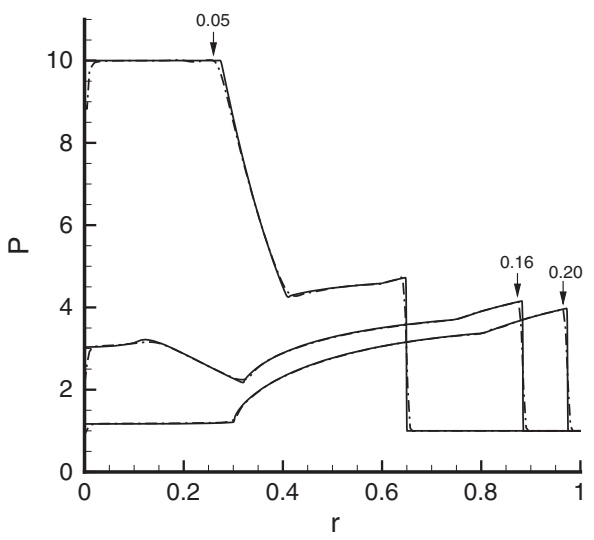

(d)

Figure 2. Density isolines for the explosion problem with cylindrical symmetry solved in the polar plane $R-\theta$ (a) $t=0.05$, (b) $t=0.16$ and (c) $t=0.20$. Every isoline corresponds to a difference of $\Delta \rho / \rho_{\text {ref }}=0.03$. In (d), the pressure signal, computed from the two-dimensional solution (dashed line), is reported with respect to the one-dimensional cylindrical reference solution (continuous line). 


\section{NUMERICAL RESULTS}

In the present section, numerical results in two-dimensional cylindrical coordinates $(R-\theta$ and $Z-R$ ) are presented for the proposed hybrid FE/FV scheme. Two different test cases are considered, namely the explosion and the implosion problems in Section 4.1 and the interaction between converging shocks waves and cylindrical and spherical obstacles in Section 4.2. The space and temporal convergence of the hybrid FE/FV schemes is assessed in Section 4.1.1.

\subsection{Numerical simulation of the explosion and implosion problems with cylindrical and spherical symmetry}

The explosion and implosion problems are special cases of the Riemann problem of gasdynamics, in which the initial solution is either cylindrically or spherically symmetric and discontinuous at a given distance from the origin. The discontinuity separates two constant states, see, for example, $[3,4]$. Flows with such initial conditions arise, for instance, in the case of an abrupt release of energy by means of cylindrical or spherical explosive charges or a focused laser beam (explosion problem) or in the context of ICF or nuclear devices (implosion problem).

Assuming that the characteristic time for energy release is negligible with respect to the one of the flow, the fluid velocity is zero everywhere, and from mass conservation, the density is constant in the whole flow field. The initial conditions therefore read

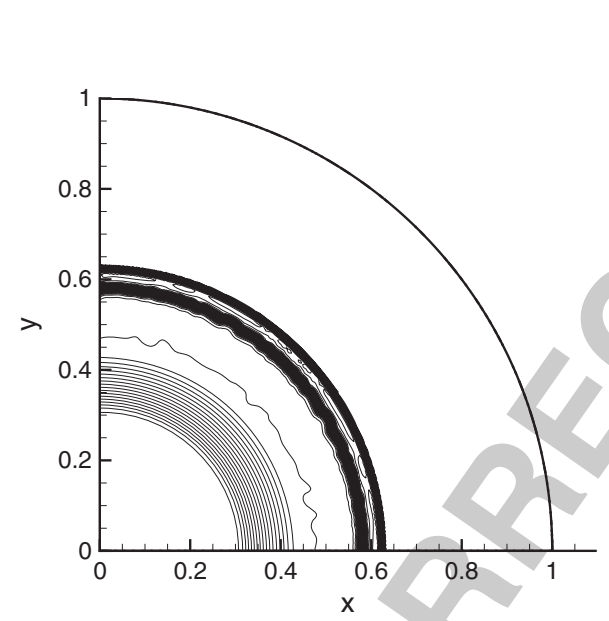

(a)

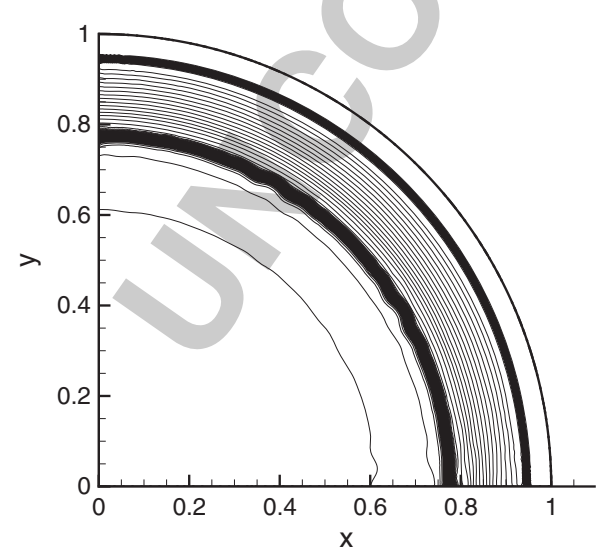

(c)

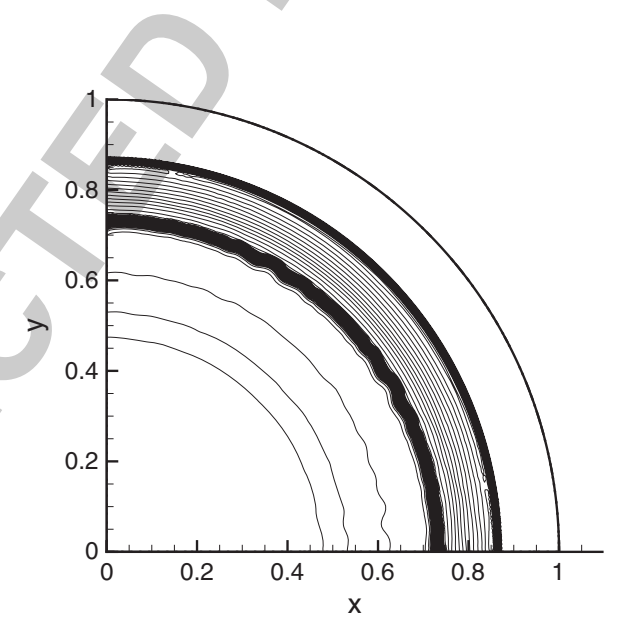

(b)

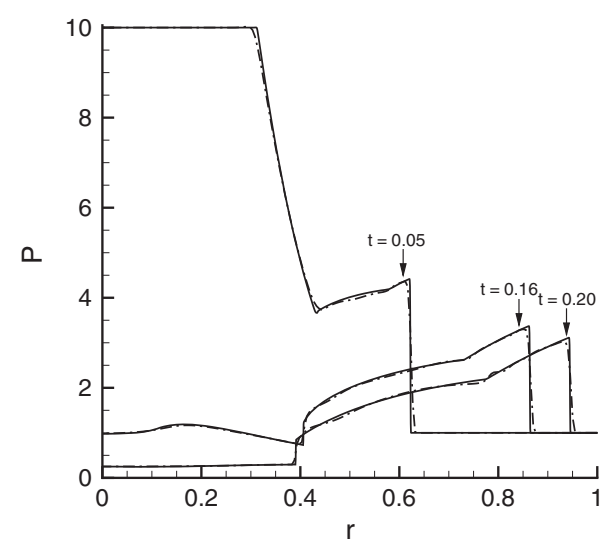

(d)

Figure 3. Density isolines for the explosion problem with spherical symmetry solved in the axisymmetric plane $Z-R$ (a) $t=0.05$, (b) $t=0.16$ and (c) $t=0.20$. Every isoline corresponds to a difference of $\Delta \rho / \rho_{\text {ref }}=0.03$. In (d), the pressure signal, computed from the two-dimensional solution (dashed line), is reported with respect to the one-dimensional cylindrical reference solution (continuous line). 


$$
\rho(R)=\rho_{1} \quad \forall R, \quad u(R)=0 \quad \forall R, \quad P(R)= \begin{cases}P_{1} & \text { for } R \leqslant R_{i} \\ P_{2} & \text { for } R>R_{i}\end{cases}
$$

with $u=m / \rho$ fluid velocity. For the explosion and implosion problem, one has $P_{1}>P_{2}$ and $P_{1}<P_{2}$, respectively. For all the numerical tests, the dimensionless position of the initial discontinuity is $R_{i}=0.5$. All variables are made dimensionless with respect to an arbitrary reference state, indicated with the subscript ref as follows:

$$
\bar{x}=x \bar{L}_{\text {ref },} \quad \bar{t}=t \bar{L}_{\text {ref }} \sqrt{\bar{\rho}_{\text {ref }} / \bar{P}_{\text {ref }}}, \quad \bar{\rho}=\rho \bar{\rho}_{\text {ref }}, \quad \bar{u}=u \sqrt{\bar{P}_{\text {ref }} / \bar{\rho}_{\text {ref }}}, \quad \bar{P}=P \bar{P}_{\text {ref }},
$$

where the overbar indicates dimensional quantities and where $\bar{L}_{\text {ref }}$ is the reference length. In all cases, the reference length is the radius of the computational domain, which encompasses 90 degrees of a circle centred at the origin (Figures 2-5). Reference fluid quantities are those of the unperturbed state, that is, state 2 and 1 for the explosion and implosion problem, respectively.

Figure 2(a)-(c) show the density isolines at different time on the $R-\theta$ plane for the explosion problem with cylindrical symmetry with $P_{1} / P_{2}=10$. In the explosion problem, a shock wave moves towards the external part of the domain followed by a contact discontinuity, while a rarefaction wave moves inward until it is reflected at the origin. Wall boundary conditions (no-slip

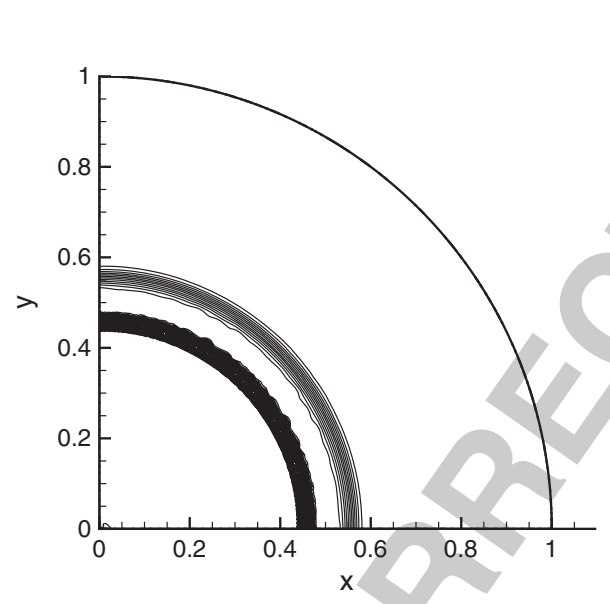

(a)

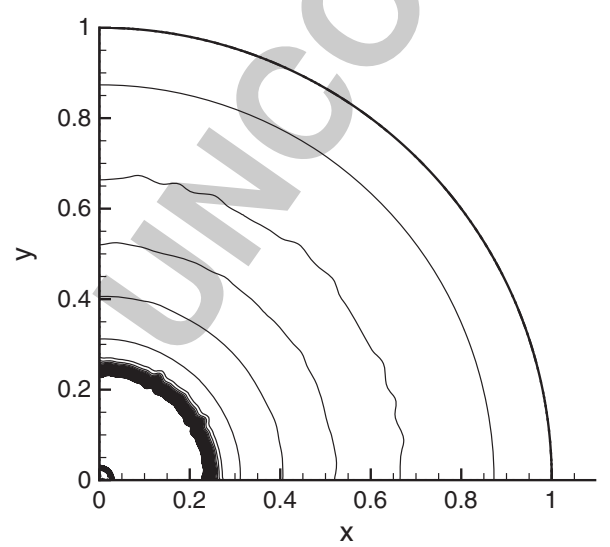

(c)

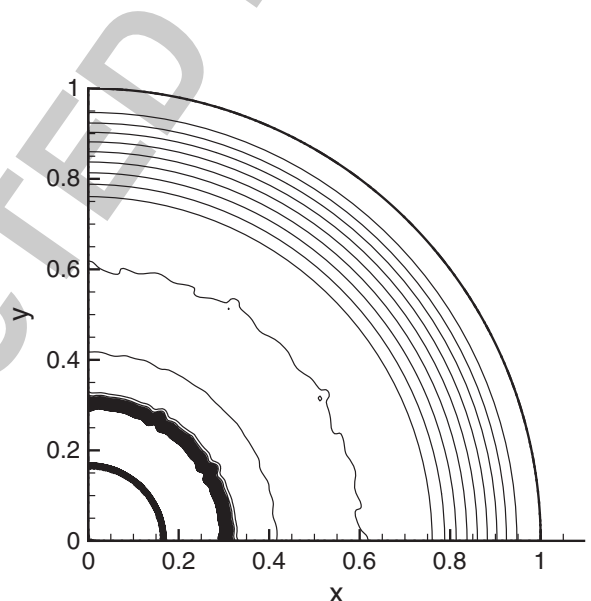

(b)

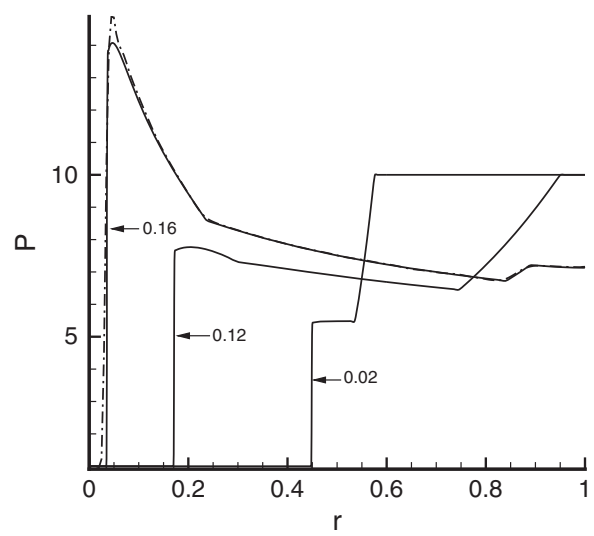

(d)

Figure 4. Density isolines for the implosion problem with cylindrical symmetry solved in the polar plane $R-\theta$ (a) $t=0.02$, (b) $t=0.12$ and (c) $t=0.16$. Every isoline corresponds to a difference of $\Delta \rho / \rho_{\text {ref }}=0.03$. In (d), the pressure signal, computed from the two-dimensional solution (dashed line), is reported with respect to the one-dimensional cylindrical reference solution (continuous line). 


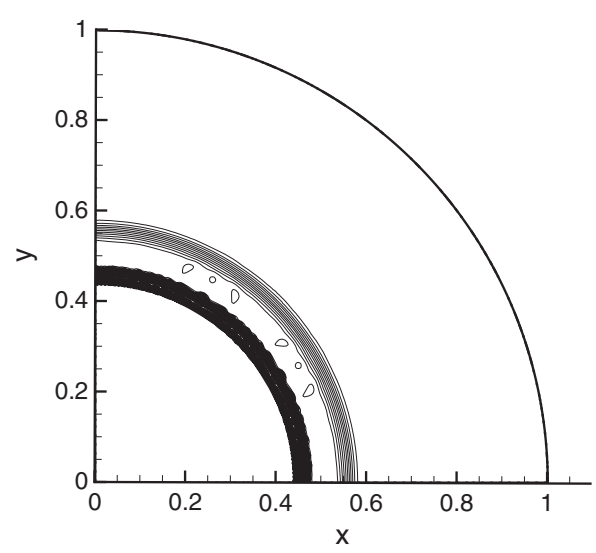

(a)

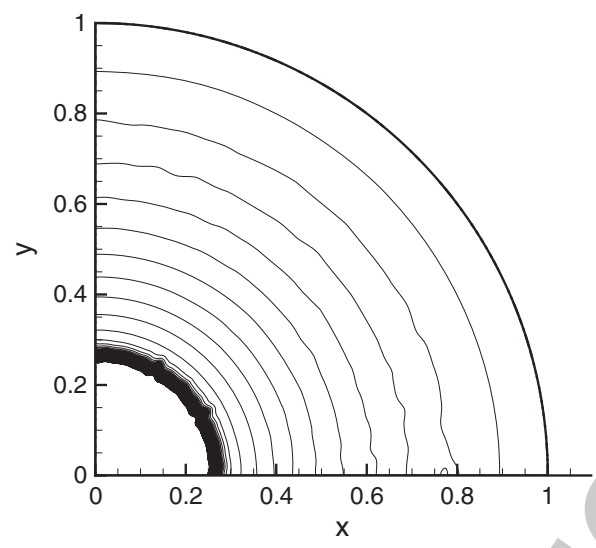

(c)

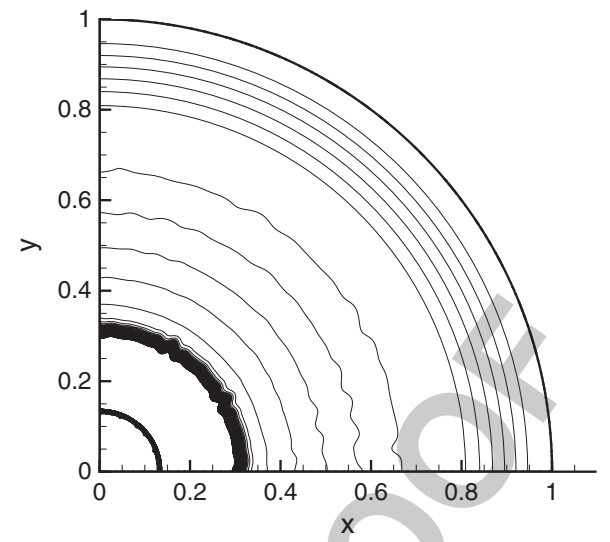

(b)

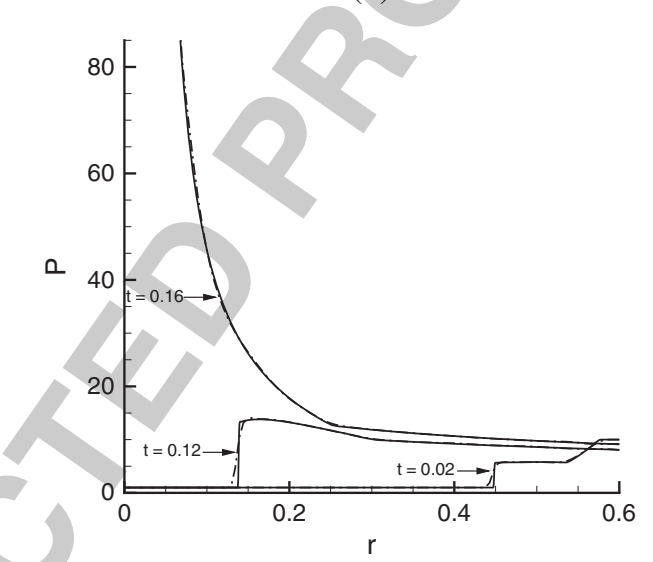

(d)

Figure 5. Density isolines for the implosion problem with spherical symmetry solved in the axisymmetrical plane $Z-R$ (a) $t=0.02$, (b) $t=0.12$ and (c) $t=0.16$. Every isoline corresponds to a difference of $\Delta \rho / \rho_{\text {ref }}=0.03$. In (d), the pressure signal, computed from the two-dimensional solution (dashed line), is reported with respect to the one-dimensional cylindrical reference solution (continuous line).

conditions) are imposed at all the boundaries of the domain. The pressure signal along the $y=0$ boundary is compared in Figure 2(d) to the reference one-dimensional solution, which was computed over a very fine grid of 2001 nodes $\left(\Delta R=5 \times 10^{-5}, \Delta t=3 \times 10^{-5}\right)$. The same initial conditions are used for the explosion problem with spherical symmetry, which is solved in the $Z-R$ plane. The density isolines, for three different time instant, are reported in Figure 3(a)-(c); the pressure signal is compared with the one-dimensional spherical solution in Figure 3(d).

The implosion problem was solved for cylindrically and spherically symmetric initial conditions in the cylindrical and axisymmetrical references, respectively, with $P_{1} / P_{2}=0.1$. In the implosion problem, a rarefaction wave moves outward, and a shock wave propagates inward. The intensity of the shock waves increases moving closer to the origin creating a high pressure and temperature region. Then, the shock waves reach the origin axis and reflects starting to move outward. A new compression is then observed propagating from the origin, which further increases both pressure and temperature of the fluid. The density isolines on the cylindrical plane, for three different time instants, are reported in Figure 4(a)-(c); the comparison of the pressure signal along the $y=0$ axis is reported in Figure 4(d). In Figure 5(a)-(c), density isolines are reported for the axisymmetrical case. Also, in this case, the pressure signal is reported and compared with the one-dimensional reference solution in Figure 5(d).

All the numerical simulations have been carried out on unstructured meshes made of triangular elements of constant size. In Figure 6, an exemplary unstructured grid is shown, which was gener- 

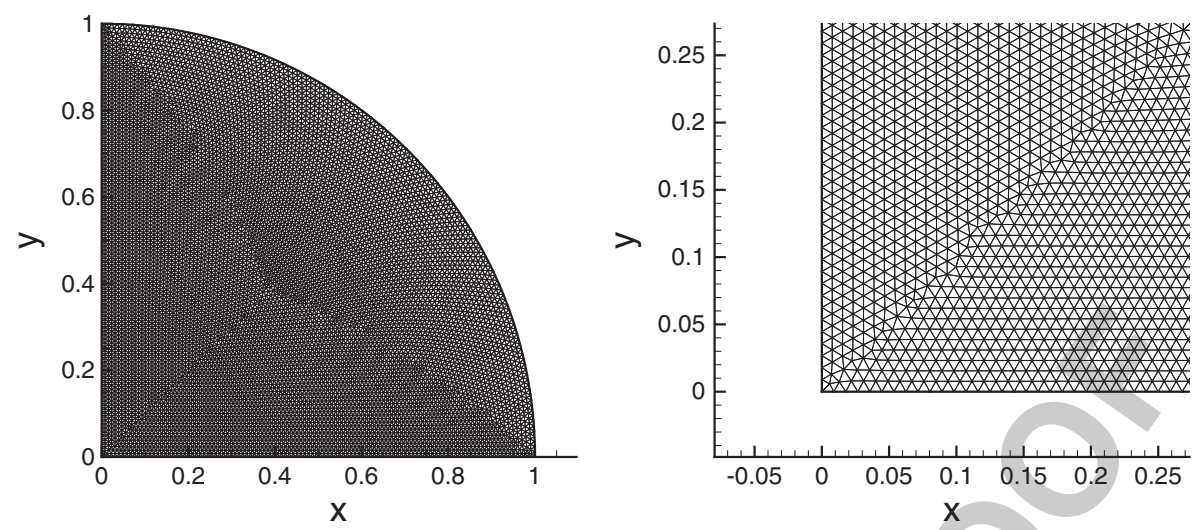

Figure 6. Coarse grid employed in the simulations. Left: entire domain. Right: enlargement close to the origin.

Table IV. Mesh employed for the explosion and implosion simulations.

\begin{tabular}{cccc}
\hline Mesh & Nodes & Triangles & Resolution \\
\hline Coarse & 9551 & 18745 & 0.01 \\
Medium & 20683 & 40841 & 0.007 \\
Fine & 39153 & 77587 & 0.005 \\
\hline
\end{tabular}

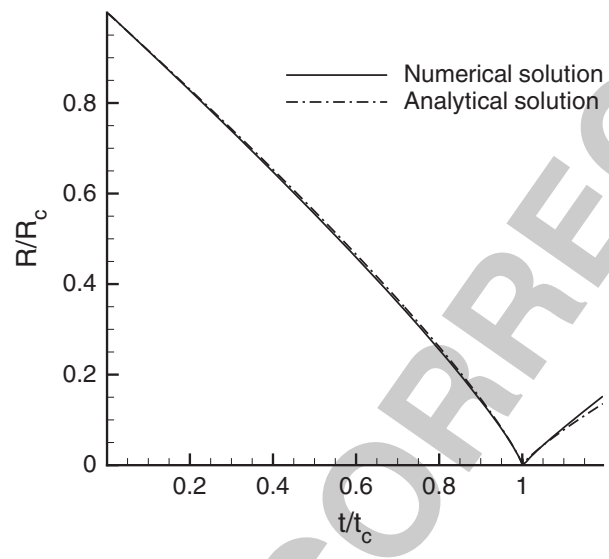

(a)

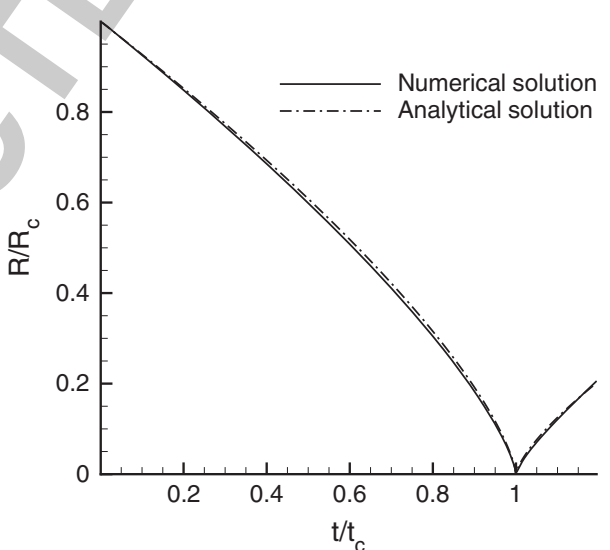

(b)

Figure 7. Radial position of the shock, in the cylindrical (a) and spherical (b) cases, for the implosion problem compared with the analytical solution of Guderley [33].

ated using an advancing-front/Delaunay technique [32]. Three different spatial resolutions for the meshes have been employed; all the details are reported in Table IV. Simulations in Figures 2-5 were carried out over the fine mesh (Table IV) with a temporal step of $2.5 \times 10^{-4}$ for the polar case and $1.5 \times 10^{-4}$ for the axisymmetrical case are reported. The CFL number is approximately 0.5 for both the cases. In all computed solutions, the wave fronts present corrugations due to the representation of the initial condition by means of a non-structured mesh, cf. Figure 6 .

4.1.1. Assessement of space and time convergence. The dependence of the numerical results presented previously on the grid spacing and on the time step is now assessed against reference one-dimensional results computed over a fine grid. To this purpose, the one-dimensional versions of the scheme, presented in details in [21], are first evaluated against the analytical reference solution 


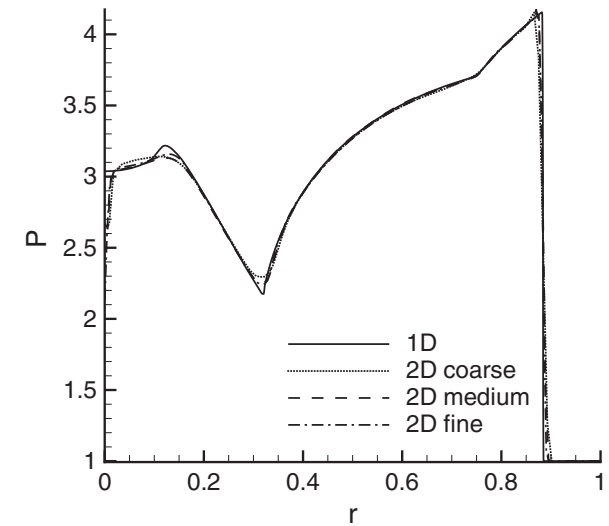

(a)

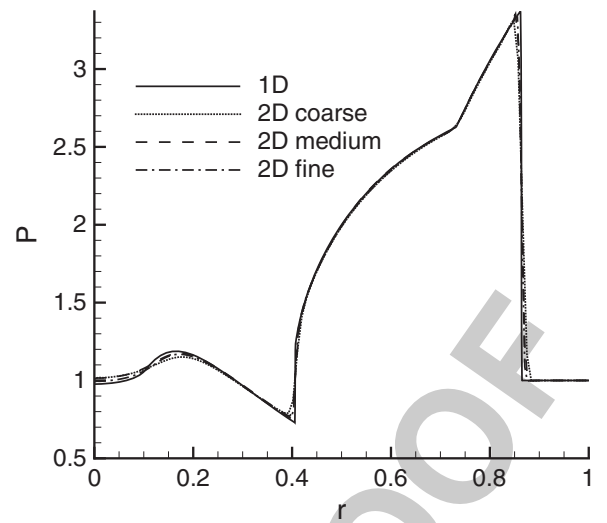

(b)

Figure 8. Pressure signal along $y=0$ for the explosion problem at $t=0.16$ with different spatial resolutions. The result is shown with respect to the one-dimensional solution. The solutions on the $R-\theta$ (a) and $Z-R$ (b) are reported.

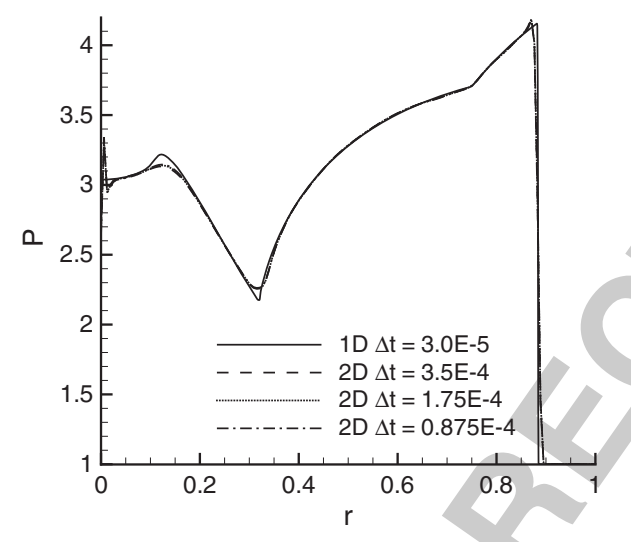

(a)

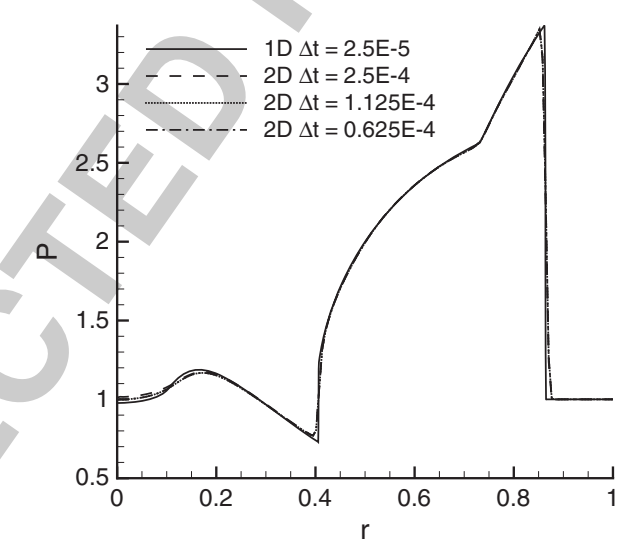

(b)

Figure 9. Pressure signal along $y=0$ for the explosion problem at $t=0.16$ with different time steps. The result is shown with respect to the one-dimensional solution. The solutions on the $R-\theta$ (a) and $Z-R$ (b).
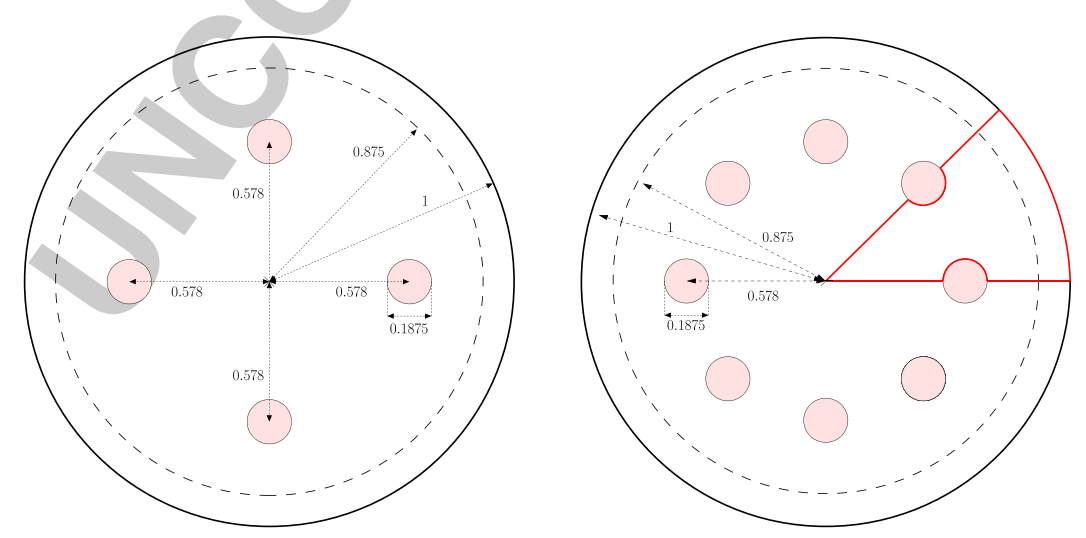

Figure 10. Computational domains used for the simulation of a cylindrical shock wave converging on four (left) and eight (right) obstacles. The dashed circumference represents the position of the discontinuity at the initial time. The red line in the eight-obstacles configuration represents the boundaries of the effective domain used for the numerical simulation. 
due to Guderley [33] for the motion of a converging shock wave in both the cylindrical $(R-\theta)$ and spherical $(Z-R)$ symmetric cases. Guderley showed that the radial position $R_{\mathrm{s}}(t)$ of the convergent shock follows the similarity law

$$
\frac{R_{\mathrm{s}}(t)}{R_{\mathrm{i}}}=A^{ \pm}\left[1-\frac{t}{t_{0}}\right]^{\alpha}, \quad \text { with } \quad A^{ \pm}=\left\{\begin{array}{l}
A^{+} \text {for } t<t_{0} \\
A^{-} \text {for } t \geq t_{0}
\end{array}\right.
$$

where $t_{0}$ is the time instant in which the shock reaches the origin and where $A^{+}, A^{-}$and $\alpha$ are constant coefficients whose values depend on the type of symmetry of the shock wave. For the ideal polytropic gases of interest here, $\alpha=0.834$ and $\alpha=0.717$ for the cylindrical and spherical symmetric cases, respectively. The coefficient $A^{+}$can be computed exactly and $A^{+}=1$ in both cylindrical or spherical symmetry. On the contrary, for coefficient $A^{-}$, only numerical approximations are available: $A^{-}=0.533$ for the cylindrical case and $A^{-}=0.653$ for the spherical one [34].

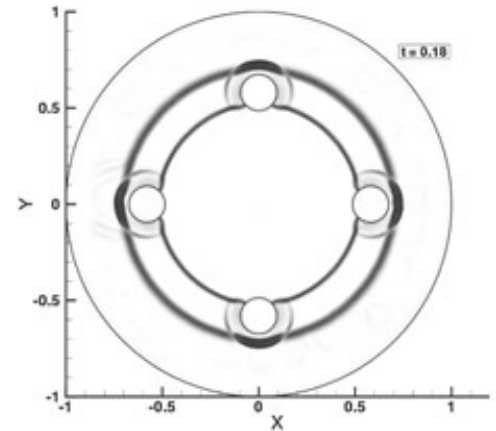

(a)

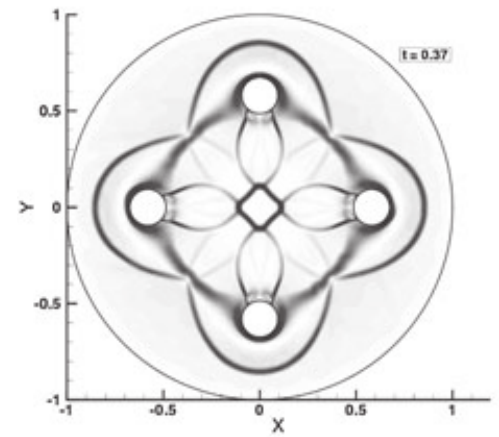

(c)

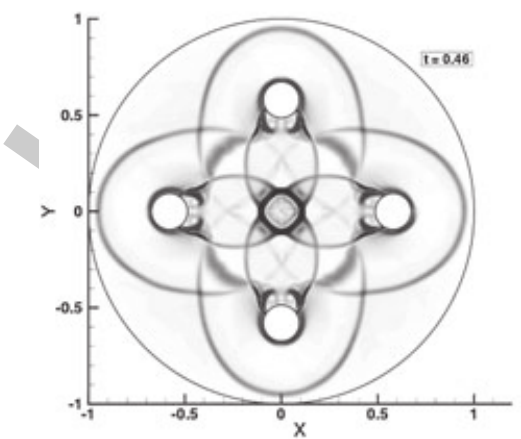

(e)

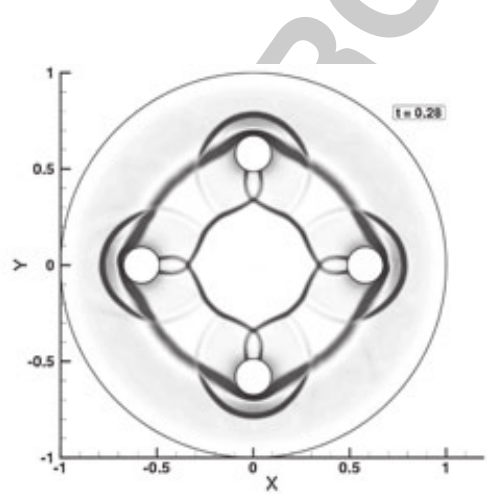

(b)

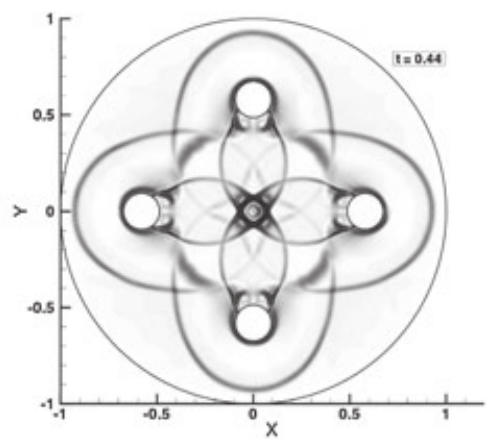

(d)

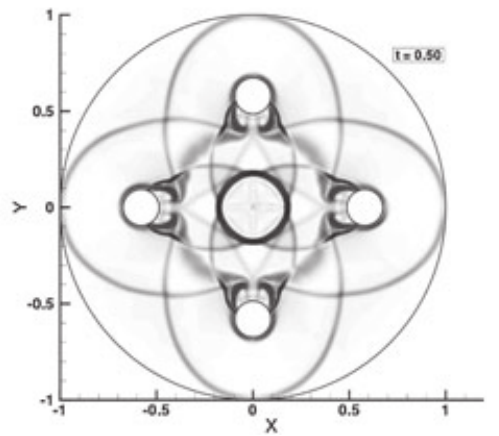

(f)

Figure 11. Numerical Schlieren of the implosion problem in cylindrical coordinates with four obstacles at different time instants. The Figures (a)-(c) show the shock before it reaches the central point of the domain; the Figures (d)-(f) show the shock after the reflection. 


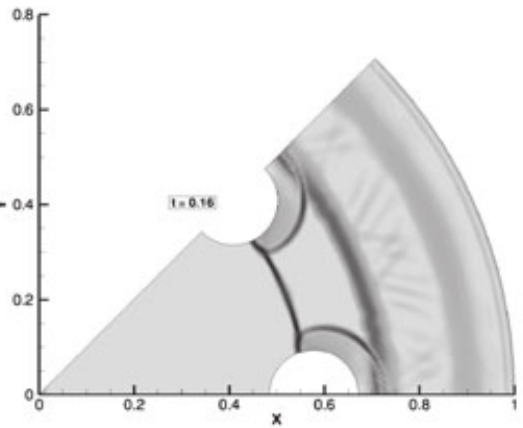

(a)

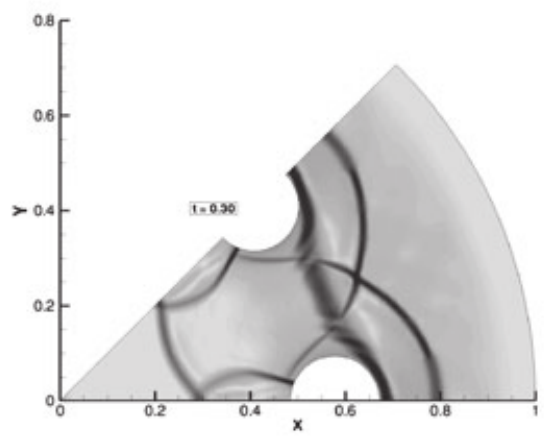

(c)

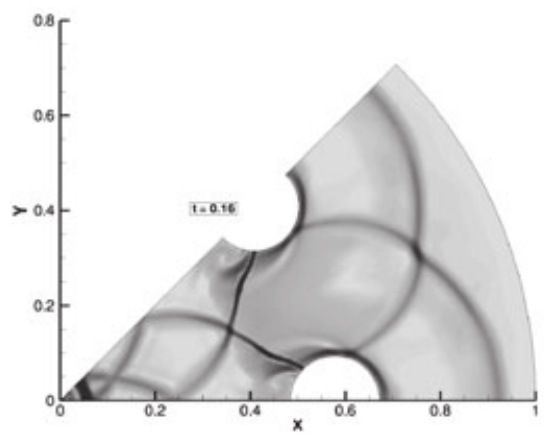

(e)

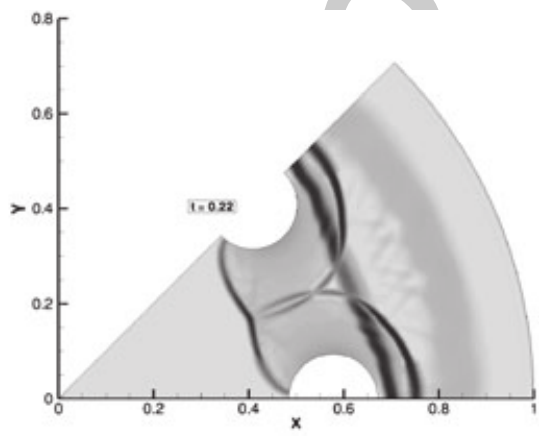

(b)

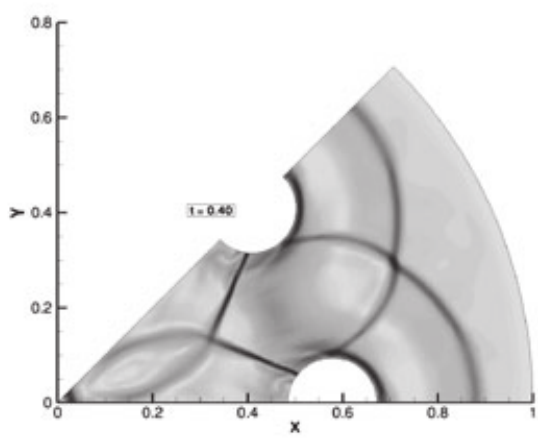

(d)

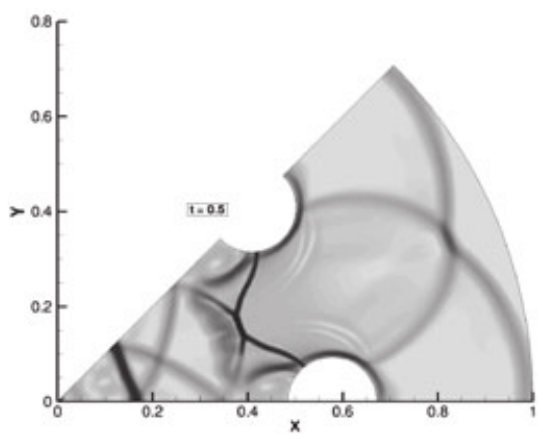

(f)

Figure 12. Numerical Schlieren of the implosion problem in cylindrical coordinates with eight obstacles at different time instants. The Figures (a)-(c) show the shock before it reaches the central point of the domain; the Figures (d)-(f) show the shock after the reflection. 
meshes (Table IV) is compared with reference one-dimensional solutions over the domain $[0,1]$ with 2001 nodes $\left(\Delta R=5 \times 10^{-4}, \Delta t=3 \times 10^{-5}\right)$ at $t=0.16$. Both the polar $R-\theta$ and the axisymmetrical solution $Z-R$ are found to be almost independent on the mesh spacing.

In Figure 9, the reference one-dimensional solutions are compared with two-dimensional computations in both the polar $R-\theta$ and axisymmetric $Z-R$ plane over the fine mesh for three different time steps, that is, $\Delta t=7 \times(1 / 2,1 / 4,1 / 8) \times 10^{-4}$. Two-dimensional solutions are found to be almost independent from the time step, and differences are apparent only at the shock location and at the origin. In this respect, it is to be noted that in all the computations in the $R-\theta$ plane, the solution at the grid node located at the origin of the reference system suffers from a significant undershoot, which however does not propagate inside the domain and does not affect the correct propagation of the reflected waves. This is believed to be related to the notorious wall heating effect pointed out in [35]. In the axisymmetric case, this local loss of monotonicity is not observed.

\subsection{Numerical simulations of shock-obstacles interaction}

Corrugation instabilities have been observed in converging shocks waves, which limit the possibility of observing a symmetric shock front, as envisaged in, for example, ICF applications [36-38]. In order to produce a symmetric and repeatable shock front, Eliasson et al. proposed to introduce in the flow field artificial disturbances arranged in a symmetric pattern [39-41]. By using a number of obstacles, it is possible to create polygonal shock fronts which are stable, with number of sides proportional to the number of obstacles. Unfortunately, the presence of obstacles along the shock path reduces the peak of temperature and pressure at the shock focus point, and therefore, it is of paramount importance to choice the shape and arrangement of the obstacles so to minimize losses.

Shock reshaping by means of cylindrical obstacles is simulated here in cylindrical coordinates $(R-$ $\theta$ plane) by applying the proposed scheme to the propagation of cylindrical converging shock waves encountering symmetric patterns of cylindrical obstacles. One four-obstacle and one eight-obstacle configurations are considered, as shown in Figure 10.

In the case of the four-obstacle configuration, the entire circular domain is considered, so to assess the scheme capabilities to preserve the symmetries of the shock structure. The domain is discretized by an unstructured grid made of 36713 nodes and 72612 triangles. For the configuration with eight obstacles, the problem symmetry is used to reduce the size of the computational domain, as shown in Figure (10). The computational domain encompasses a circular arc of 45 degrees; the computational mesh is made of 11112 nodes and 21978 triangles. In all simulations, a CFL number 0.5 is used. Initial conditions for both cases correspond to a pressure and density discontinuity- the socalled shock-tube problem-at $R=0.875$, with a pressure and density ratio of 10 across the initial discontinuity.
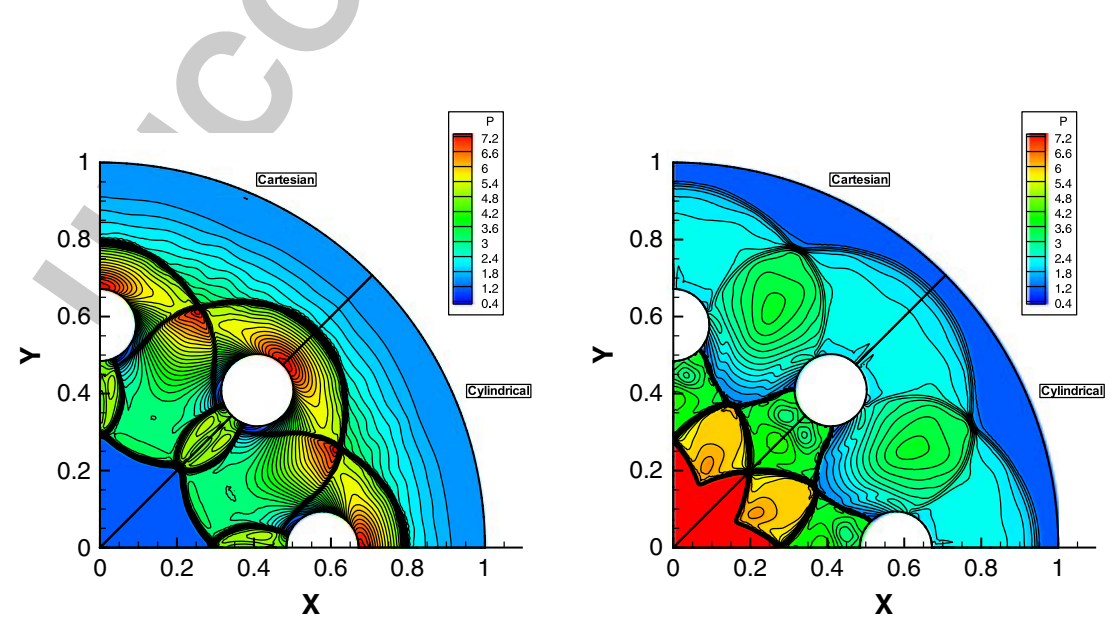

Figure 13. Comparison between the solutions of the simulations in Cartesian and cylindrical coordinates systems for the problem with eight obstacles before (left) and after (right) the shock reflection at the centre of the domain. 
In Figure 11, the numerical Schlieren of the four-obstacle problem is showed at different time levels. The numerical scheme is able to reproduce the main features of the problem observed in experimental results, which are discussed in details in [41]. At time $t=0.18$, Figure 11(a), the converging shock wave is almost past the obstacles. A cylindrical reflected shock moving outward surrounds each obstacle and interacts with the incoming contact discontinuity. At $t=0.28$, Figure 11(b), the converging shock wave exhibits an approximately octagonal shape, with sides slightly curved outward. At time $t=0.37$, a quadrilateral shape is observed, with vertexes aligned to the obstacle positions. At later times, the shock reaches the origin, and it is reflected in the outward direction, as shown in Figure 11(c)-(f). In Figure 12, a similar behaviour can be observed the octagonal shock shape produced with eight cylindrical obstacles, where in this case, the resulting polygonal shock has eight sides.

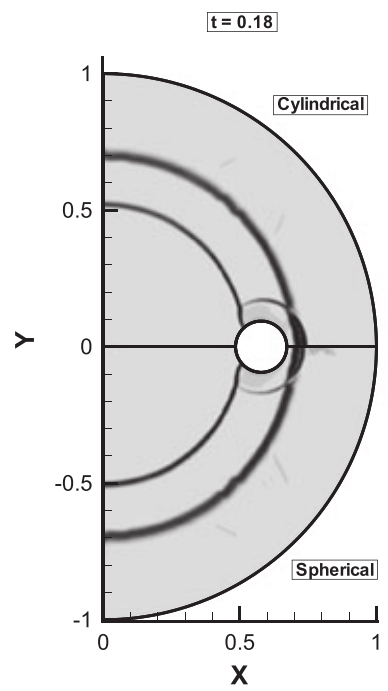

(a)

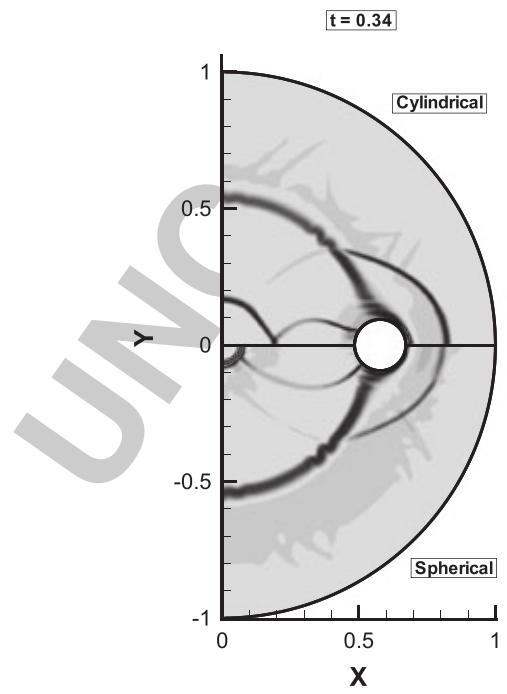

(c)

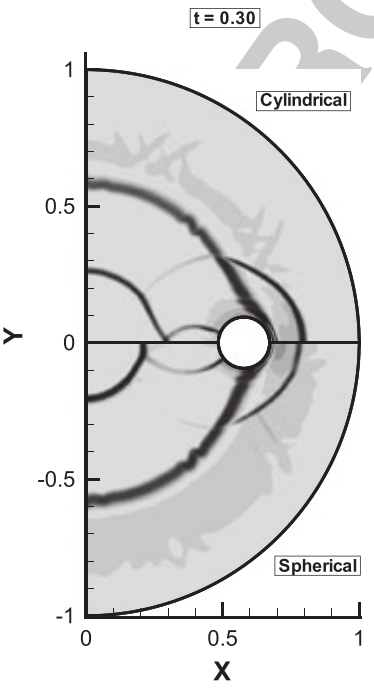

(b)

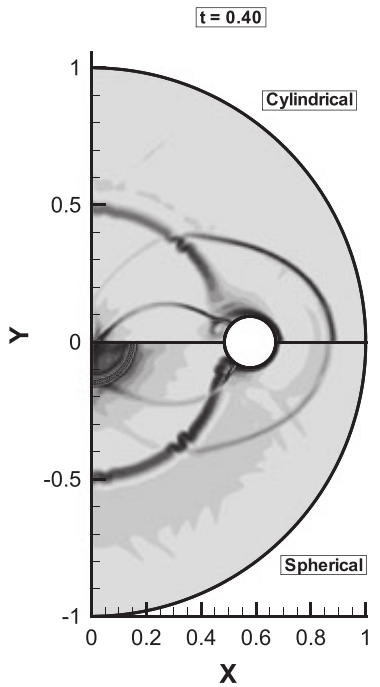

(d)

Figure 14. Numerical Schlieren of the implosion problem for a single obstacle in a cylindrical (top) and spherical (bottem) coordinates system. The cylindrical problem describes the interaction of a cylindrical shock wave with a cylindrical obstacles, the spherical one that of a spherical shock wave with a spherical obstacle. 
The results of the numerical simulations obtained in the cylindrical reference are compared with the corresponding simulations in Cartesian coordinates. In the case of the more complex shock structure in the eight-obstacle configuration, a very good match between the solutions in the cylindrical and Cartesian reference systems can be seen in Figure 13, before and after the shock reflection at the centre.

To conclude, the flow field generated by the interaction of a cylindrical converging shock wave with a cylindrical obstacle is compared with that observed during the interaction of a spherical converging shock with a spherical obstacle. In both cases, a single obstacle is considered, whose geometry and distance from the origin is as in Figure 10. In both the cylindrical cases (described in the $R-\theta$ plane) and the spherical one ( $Z-R$ plane), the computational mesh is made of 22649 nodes and 44781 triangles. In the case of the simulation on the $Z-R$ plane, the axis $Z$ is aligned with horizontal axis. Figure 14 reports the comparison between the cylindrical and spherical shock waves at different time levels. In each plot, the upper portion of the domain corresponds to the cylindrical geometry, the lower portion to the spherical one. In the cylindrical case, the interaction between the shock and the obstacle results in the formation of a planar portion of the shock wave. Not surprisingly, this is not the case for the spherical obstacle, in which the modification of the shock geometry is less relevant. This is possibly due to the limited area ratio of the obstacle frontal area and that of the propagating shock waves [42].

\section{CONCLUSIONS}

A consistent hybrid FE/FV scheme was proposed to solve conservation laws in a three-dimensional cylindrical reference. Consistency between the mass-lumped Bubnov-Galerkin FE scheme for Lagrangian linear elements and the node-centred FV schemes was achieved by devising equivalence conditions relating the FV metrics to the corresponding FE integrals, under the condition that the discrete representation of all relevant differential operators is the same for both the FE and the FE schemes. Differently from the equivalence conditions presented in $[17,18]$ for Cartesian coordinates and in [19] for cylindrical axisymmetrical, equivalence conditions for a three-dimensional cylindrical coordinates reference system were derived here for first time without introducing any approximation into the FE discrete expression of the divergence operator. The two-dimensional equivalence conditions for the polar and the axisymmetrical cases are also explicitly derived.

A high-resolution TVD consistent hybrid FV/FE schemes was proposed for solving the compressible Euler equations for in viscid flows in a cylindrical reference based on the proposed equivalence conditions. Numerical results obtained with the new solver compare fairly well with one-dimensional simulations and simplified models.

\section{APPENDIX A: NODE-PAIR FORM OF THE FINITE ELEMENT METHOD IN CYLINDRICAL} COORDINATES

In this Appendix, the derivation of the node-pair representation (12) of the FE discrete problem (11) is detailed.

First, the following identity,

$$
\begin{aligned}
2 \sum_{k \in \mathcal{N}_{i}} \boldsymbol{f}_{k}^{\varnothing} \cdot \int_{\Omega_{i k}} R \varphi_{k} \nabla^{\varnothing} \varphi_{i} \mathrm{~d} \Omega^{\varnothing}= & -\sum_{k \in \mathcal{N}_{i, \neq}}\left[\left(f_{k}^{\varnothing}+\boldsymbol{f}_{i}^{\varnothing}\right) \cdot \eta_{i k}^{\varnothing}+\left(\boldsymbol{f}_{k}^{\varnothing}-\boldsymbol{f}_{i}^{\varnothing}\right) \cdot \zeta_{i k}^{\varnothing}\right] \\
& +\sum_{k \in \mathcal{N}_{i, \neq}^{\partial}}\left(\boldsymbol{f}_{k}^{\varnothing}-\boldsymbol{f}_{i}^{\varnothing}\right) \cdot \int_{\partial \Omega_{i k}^{\partial}} R \varphi_{i} \varphi_{k} \boldsymbol{n}^{\varnothing} \mathrm{d} \Sigma^{\varnothing}
\end{aligned}
$$

is shown to hold. To this purpose, the integral in the left-hand side is written as the sum of contributions from each element $e$ of the mesh as follows:

$$
\sum_{k \in \mathcal{N}_{i}} \boldsymbol{f}_{k}^{\varnothing} \cdot \int_{\Omega_{i k}} R \varphi_{k} \nabla^{\varnothing} \varphi_{i} \mathrm{~d} \Omega^{\varnothing}=\sum_{e \in \mathcal{E}_{i}} \sum_{k \in \mathcal{N}^{e}} \boldsymbol{f}_{k}^{\varnothing} \cdot \int_{\Omega^{e}} R \varphi_{k} \nabla^{\varnothing} \varphi_{i} \mathrm{~d} \Omega^{\varnothing},
$$


where $\mathcal{E}_{i}$ is the set of the element which shares the node $i$ and $\mathcal{N}^{e}$ is the set of the nodes of the element $e$. In the first summation, only those elements that belong to the support $\Omega_{i}=\bigcup_{e \in \mathcal{E}_{i}} \Omega^{e}$ of the node $i$ generate a non-null contribution. Considering the following identity provided by the Gauss theorem,

$$
\int_{\Omega^{e}} \nabla^{\varnothing}\left(R \varphi_{i} \varphi_{k}\right) \mathrm{d} \Omega^{\varnothing}=\int_{\partial \Omega^{e}} R \varphi_{i} \varphi_{k} n^{\varnothing} \mathrm{d} \Sigma^{\varnothing}
$$

it is possible to write

$$
\int_{\Omega^{e}} R \varphi_{k} \nabla^{\varnothing} \varphi_{i} \mathrm{~d} \Omega^{\varnothing}=-\int_{\Omega^{e}} R \varphi_{i} \nabla^{\varnothing} \varphi_{k} \mathrm{~d} \Omega^{\varnothing}-\int_{\Omega^{e}} \varphi_{i} \varphi_{k} \nabla^{\varnothing} R \mathrm{~d} \Omega^{\varnothing}+\int_{\partial \Omega^{e}} R \varphi_{i} \varphi_{k} n^{\varnothing} \mathrm{d} \Sigma^{\varnothing},
$$

and therefore,

$$
\begin{aligned}
2 \int_{\Omega^{e}} R \varphi_{k} \nabla^{\varnothing} \varphi_{i} \mathrm{~d} \Omega^{\varnothing}= & \int_{\Omega^{e}} R \varphi_{k} \nabla^{\varnothing} \varphi_{i} \mathrm{~d} \Omega^{\varnothing}+\int_{\Omega^{e}} R \varphi_{k} \nabla^{\varnothing} \varphi_{i} \mathrm{~d} \Omega^{\varnothing} \\
= & \int_{\Omega^{e}} R \varphi_{k} \nabla^{\varnothing} \varphi_{i} \mathrm{~d} \Omega^{\varnothing}-\int_{\Omega^{e}} R \varphi_{i} \nabla^{\varnothing} \varphi_{k} \mathrm{~d} \Omega^{\varnothing} \\
& \quad-\int_{\Omega^{e}} \varphi_{i} \varphi_{k} \nabla^{\varnothing} R \mathrm{~d} \Omega^{\varnothing}+\int_{\partial \Omega^{e}} R \varphi_{i} \varphi_{k} n^{\varnothing} \mathrm{d} \Sigma^{\varnothing} \\
= & -\eta_{i k}^{e, \varnothing}-\int_{\Omega^{e}} \varphi_{i} \varphi_{k} \hat{\boldsymbol{R}} \mathrm{d} \Omega^{\varnothing}+\int_{\partial \Omega^{e}} R \varphi_{i} \varphi_{k} \boldsymbol{n}^{\varnothing} \mathrm{d} \Sigma^{\varnothing},
\end{aligned}
$$

where we introduced the contribution $\eta_{i k}^{e, \varnothing}$ of the element $e$ to the vector $\boldsymbol{\eta}_{i k}^{\varnothing}$

$$
\eta_{i k}^{e, \varnothing \stackrel{\text { def }}{=}} \int_{\Omega_{i k} \cap \Omega^{e}} R\left(\varphi_{i} \nabla^{\varnothing} \varphi_{k}-\varphi_{k} \nabla^{\varnothing} \varphi_{i}\right)
$$

with $\boldsymbol{\eta}_{i k}^{\varnothing}=\sum_{e \in\left(\mathcal{E}_{i} \cap \mathcal{E}_{k}\right)} \eta_{i k}^{e, \varnothing}$ and we used $\nabla^{\varnothing} R=\hat{\boldsymbol{R}}$. Using (A.4), the integral (A.2) becomes

$2 \sum_{k \in \mathcal{N}_{i}} \boldsymbol{f}_{k}^{\varnothing} \cdot \int_{\Omega_{i k}} R \varphi_{k} \nabla^{\varnothing} \varphi_{i} \mathrm{~d} \Omega^{\varnothing}=-\sum_{e \in \mathcal{E}_{i}} \sum_{k \in \mathcal{N}^{e}} \boldsymbol{f}_{k}^{\varnothing} \cdot\left(\eta_{i k}^{e, \varnothing}+\int_{\Omega^{e}} \varphi_{i} \varphi_{k} \hat{\boldsymbol{R}} \mathrm{d} \Omega^{\varnothing}-\int_{\partial \Omega^{e}} R \varphi_{i} \varphi_{k} \boldsymbol{n}^{\varnothing} \mathrm{d} \Sigma^{\varnothing}\right)$.

It is also possible to write (A.4) as

$$
\eta_{i k}^{e, \varnothing}=-2 \int_{\Omega^{e}} R \varphi_{k} \nabla^{\varnothing} \varphi_{i} \mathrm{~d} \Omega^{\varnothing}-\int_{\Omega^{e}} \varphi_{i} \varphi_{k} \hat{\boldsymbol{R}} \mathrm{d} \Omega^{\varnothing}+\int_{\partial \Omega^{e}} R \varphi_{i} \varphi_{k} \boldsymbol{n}^{\varnothing} \mathrm{d} \Sigma^{\varnothing}
$$

and with (A.3), it can be recast

$$
\eta_{i k}^{e, \varnothing}=2 \int_{\Omega^{e}} R \varphi_{i} \nabla^{\varnothing} \varphi_{k} \mathrm{~d} \Omega^{\varnothing}+\int_{\Omega^{e}} \varphi_{i} \varphi_{k} \hat{R} \mathrm{~d} \Omega^{\varnothing}-\int_{\partial \Omega^{e}} R \varphi_{i} \varphi_{k} \boldsymbol{n}^{\varnothing} \mathrm{d} \Sigma^{\varnothing} .
$$

Summing this last relation for all nodes $k$ which belong to $\Omega^{e}$ and considering that $\sum_{k \in \mathcal{N}^{e}} \nabla^{\varnothing} \varphi_{k}\left(\boldsymbol{x}^{\varnothing}\right)=\mathbf{0}, \forall \boldsymbol{x}^{\varnothing} \in \Omega^{e}$, we obtain

$$
\sum_{k \in \mathcal{N}^{e}}\left(\eta_{i k}^{e, \varnothing}-\int_{\Omega^{e}} \varphi_{i} \varphi_{k} \hat{\boldsymbol{R}} \mathrm{d} \Omega^{\varnothing}+\int_{\partial \Omega^{e}} R \varphi_{i} \varphi_{k} \boldsymbol{n}^{\varnothing} \mathrm{d} \Sigma^{\varnothing}\right)=\mathbf{0}
$$

Summing over all the elements belong to $\mathcal{E}_{i}$ and perform the scalar product with $\boldsymbol{f}_{i}^{\varnothing}$, we obtain the following relation

$$
\sum_{e \in \mathcal{E}_{i}} \sum_{k \in \mathcal{N}^{e}} \boldsymbol{f}_{i}^{\varnothing} \cdot\left(\eta_{i k}^{e, \varnothing}-\int_{\Omega^{e}} \varphi_{i} \varphi_{k} \hat{\boldsymbol{R}} \mathrm{d} \Omega^{\varnothing}+\int_{\partial \Omega^{e}} R \varphi_{i} \varphi_{k} \boldsymbol{n}^{\varnothing} \mathrm{d} \Sigma^{\varnothing}\right)=\mathbf{0} .
$$


Subtracting the last relation from the right-hand side of (A.5), it follows

$$
\begin{aligned}
2 \sum_{k \in \mathcal{N}_{i}} \boldsymbol{f}_{k}^{\varnothing} \cdot \int_{\Omega_{i k}} R \varphi_{k} \nabla^{\varnothing} \varphi_{i} \mathrm{~d} \Omega^{\varnothing}= & -\sum_{e \in \mathcal{E}_{i}} \sum_{k \in \mathcal{N}^{e}}\left[\left(\boldsymbol{f}_{k}^{\varnothing}+\boldsymbol{f}_{i}^{\varnothing}\right) \cdot \eta_{i k}^{e, \varnothing}+\left(\boldsymbol{f}_{k}^{\varnothing}-\boldsymbol{f}_{i}^{\varnothing}\right) \cdot \int_{\Omega^{e}} \varphi_{i} \varphi_{k} \hat{\boldsymbol{R}} \mathrm{d} \Omega^{\varnothing}\right] \\
& +\sum_{e \in \mathcal{E}_{i}} \sum_{k \in \mathcal{N}^{e}}\left(\boldsymbol{f}_{k}^{\varnothing}-\boldsymbol{f}_{i}^{\varnothing}\right) \cdot \int_{\partial \Omega^{e}} R \varphi_{i} \varphi_{k} \boldsymbol{n}^{\varnothing} \mathrm{d} \Sigma^{\varnothing} .
\end{aligned}
$$

Because $\boldsymbol{\eta}_{i k}^{e, \varnothing}=\mathbf{0}$ for $e \notin\left(\mathcal{E}_{i} \cap \mathcal{E}_{k}\right), \boldsymbol{\eta}_{i k}^{\varnothing}=\sum_{e \in\left(\mathcal{E}_{i} \cap \mathcal{E}_{k}\right)} \boldsymbol{\eta}_{i k}^{e, \varnothing}$ and $\boldsymbol{\eta}_{i i}^{\varnothing}=\mathbf{0}$, one obtains

$$
\begin{aligned}
2 \sum_{k \in \mathcal{N}_{i}} \boldsymbol{f}_{k}^{\varnothing} \cdot \int_{\Omega_{i k}} R \varphi_{k} \nabla^{\varnothing} \varphi_{i} \mathrm{~d} \Omega^{\varnothing}= & -\sum_{k \in \mathcal{N}_{i, \neq}}\left[\left(\boldsymbol{f}_{k}^{\varnothing}+\boldsymbol{f}_{i}^{\varnothing}\right) \cdot \eta_{i k}^{\varnothing}+\left(\boldsymbol{f}_{k}^{\varnothing}-\boldsymbol{f}_{i}^{\varnothing}\right) \cdot \zeta_{i k}^{\varnothing}\right] \\
& +\sum_{k \in \mathcal{N}_{i, \neq}^{\partial}}\left(\boldsymbol{f}_{k}^{\varnothing}-\boldsymbol{f}_{i}^{\varnothing}\right) \cdot \int_{\partial \Omega_{i k}^{\partial}} R \varphi_{i} \varphi_{k} \boldsymbol{n}^{\varnothing} \mathrm{d} \Sigma^{\varnothing}
\end{aligned}
$$

that is the relation we are searching for.

The second identity to be used reads

$$
\sum_{k \in \mathcal{N}_{i}^{\partial}} \boldsymbol{f}_{k}^{\varnothing} \cdot \int_{\partial \Omega_{i k}^{\partial}} R \varphi_{i} \varphi_{k} \boldsymbol{n}^{\varnothing} \mathrm{d} \Sigma^{\varnothing}=\sum_{k \in \mathcal{N}_{i, \neq}^{\partial}}\left(\boldsymbol{f}_{k}^{\varnothing}-\boldsymbol{f}_{i}^{\varnothing}\right) \cdot \chi_{i k}^{\partial, \varnothing}+\boldsymbol{f}_{i}^{\varnothing} \cdot \xi_{i}^{\partial, \varnothing} .
$$

Identity (A.7) can be derived by highlighting the contribution of the node $i$ in the left side of (A.7), that is

$$
\sum_{k \in \mathcal{N}_{i}^{\partial}} \boldsymbol{f}_{k}^{\varnothing} \cdot \int_{\partial \Omega_{i k}^{\partial}} R \varphi_{i} \varphi_{k} \boldsymbol{n}^{\varnothing} \mathrm{d} \Sigma^{\varnothing}=\sum_{k \in \mathcal{N}_{i, \neq}^{\partial}} \boldsymbol{f}_{k}^{\varnothing} \cdot \int_{\partial \Omega_{i k}^{\partial}} R \varphi_{i} \varphi_{k} \boldsymbol{n}^{\varnothing} \mathrm{d} \Sigma^{\varnothing}+\boldsymbol{f}_{i}^{\varnothing} \cdot \int_{\partial \Omega_{i k}^{\partial}} R \varphi_{i} \varphi_{i} \boldsymbol{n}^{\varnothing} \mathrm{d} \Sigma^{\varnothing} .
$$

The quantity $\sum_{k \in \mathcal{N}_{i, \neq}^{\partial}} \boldsymbol{f}_{i}^{\varnothing} \cdot \int_{\partial \Omega_{i k}^{\partial}} R \varphi_{i} \varphi_{k} \boldsymbol{n}^{\varnothing} \mathrm{d} \Sigma^{\varnothing}$ is now added and subtracted to the last relation to obtain

$$
\begin{aligned}
\sum_{k \in \mathcal{N}_{i}^{\partial}} \boldsymbol{f}_{k}^{\varnothing} \cdot \int_{\partial \Omega_{i k}^{\partial}} R \varphi_{i} \varphi_{k} \boldsymbol{n}^{\varnothing} \mathrm{d} \Sigma^{\varnothing}= & \sum_{k \in \mathcal{N}_{i, \neq}^{\partial}}\left(\boldsymbol{f}_{k}^{\varnothing}-\boldsymbol{f}_{i}^{\varnothing}\right) \cdot \int_{\partial \Omega_{i k}^{\partial}} R \varphi_{i} \varphi_{k} \boldsymbol{n}^{\varnothing} \mathrm{d} \Sigma^{\varnothing} \\
& +\boldsymbol{f}_{i}^{\varnothing} \cdot \sum_{k \in \mathcal{N}_{i}^{\partial}} \int_{\partial \Omega_{i k}^{\partial}} R \varphi_{i} \varphi_{k} \boldsymbol{n}^{\varnothing} \mathrm{d} \Sigma^{\varnothing} .
\end{aligned}
$$

Recalling that $\sum_{k \in \mathcal{N}_{e}} \varphi_{k}\left(x^{\varnothing}\right)=1, x^{\varnothing} \in \Omega^{e}, \forall e \in \mathcal{E}$, one has

$$
\begin{aligned}
\sum_{k \in \mathcal{N}_{i}^{\partial}} \boldsymbol{f}_{k}^{\varnothing} \cdot \int_{\partial \Omega_{i k}^{\partial}} R \varphi_{i} \varphi_{k} \boldsymbol{n}^{\varnothing} \mathrm{d} \Sigma^{\varnothing} & =\sum_{k \in \mathcal{N}_{i, \neq}^{\partial}}\left(\boldsymbol{f}_{k}^{\varnothing}-\boldsymbol{f}_{i}^{\varnothing}\right) \cdot \int_{\partial \Omega_{i k}^{\partial}} R \varphi_{i} \varphi_{k} \boldsymbol{n}^{\varnothing} \mathrm{d} \Sigma^{\varnothing}+\boldsymbol{f}_{i}^{\varnothing} \cdot \int_{\partial \Omega_{i}^{\partial}} R \varphi_{i} \boldsymbol{n}^{\varnothing} \mathrm{d} \Sigma^{\varnothing} \\
& =\sum_{k \in \mathcal{N}_{i, \neq}^{\partial}}\left(\boldsymbol{f}_{k}^{\varnothing}-\boldsymbol{f}_{i}^{\varnothing}\right) \cdot \chi_{i k}^{\partial, \varnothing}+\boldsymbol{f}_{i}^{\varnothing} \cdot \xi_{i}^{\partial, \varnothing},
\end{aligned}
$$

which is relation (A.7).

Finally, by making use of the identities (A.1) and (A.7), the FE form (11) can be recasted as

$$
\begin{aligned}
\sum_{k \in \mathcal{N}_{i}} M_{i k}^{\varnothing} \frac{\mathrm{d} u_{k}}{\mathrm{~d} t}= & -\sum_{k \in \mathcal{N}_{i}, \neq}\left(\frac{\boldsymbol{f}_{k}^{\varnothing}+\boldsymbol{f}_{i}^{\varnothing}}{2} \cdot \eta_{i k}^{\varnothing}+\frac{\boldsymbol{f}_{k}^{\varnothing}-\boldsymbol{f}_{i}^{\varnothing}}{2} \cdot \zeta_{i k}^{\varnothing}\right)+\sum_{k \in \mathcal{N}_{i}} \boldsymbol{f}_{k}^{\varnothing} \cdot \int_{\Omega_{i k}} \varphi_{i} \varphi_{k} \hat{\boldsymbol{R}} \mathrm{d} \Omega^{\varnothing} \\
& -\sum_{k \in \mathcal{N}_{i, \neq}^{\partial}} \frac{\boldsymbol{f}_{k}^{\varnothing}-\boldsymbol{f}_{i}^{\varnothing}}{2} \cdot \chi_{i k}^{\partial, \varnothing}-\boldsymbol{f}_{i}^{\varnothing} \cdot \xi_{i}^{\partial, \varnothing} .
\end{aligned}
$$


Moreover, because

$$
\sum_{k \in \mathcal{N}_{i}} \boldsymbol{f}_{k}^{\varnothing} \cdot \int_{\Omega_{i k}} \varphi_{i} \varphi_{k} \hat{\boldsymbol{R}} \mathrm{d} \Omega^{\varnothing}=\sum_{k \in \mathcal{N}_{i, \neq}} \boldsymbol{f}_{k}^{\varnothing} \cdot \int_{\Omega_{i k}} \varphi_{i} \varphi_{k} \hat{\boldsymbol{R}} \mathrm{d} \Omega^{\varnothing}+\boldsymbol{f}_{i}^{\varnothing} \cdot \int_{\Omega_{i k}} \varphi_{i} \varphi_{k} \hat{\boldsymbol{R}} \mathrm{d} \Omega^{\varnothing},
$$

by adding and subtracting the quantity $\sum_{k \in \mathcal{N}_{i, \neq}} f_{i}^{\varnothing} \cdot \int_{\Omega_{i k}} \varphi_{i} \varphi_{k} \hat{\boldsymbol{R}} \mathrm{d} \Omega^{\varnothing}$, one also obtains

$$
\begin{aligned}
\sum_{k \in \mathcal{N}_{i}} \boldsymbol{f}_{k}^{\varnothing} \cdot \int_{\Omega_{i k}} \varphi_{i} \varphi_{k} \hat{\boldsymbol{R}} \mathrm{d} \Omega^{\varnothing} & =\sum_{k \in \mathcal{N}_{i, \neq}}\left(\boldsymbol{f}_{k}^{\varnothing}-\boldsymbol{f}_{i}^{\varnothing}\right) \cdot \int_{\Omega_{i k}} \varphi_{i} \varphi_{k} \hat{\boldsymbol{R}} \mathrm{d} \Omega^{\varnothing}+\sum_{k \in \mathcal{N}_{i}} \boldsymbol{f}_{i}^{\varnothing} \cdot \int_{\Omega_{i k}} \varphi_{i} \varphi_{k} \hat{\boldsymbol{R}} \mathrm{d} \Omega^{\varnothing} \\
& =\sum_{k \in \mathcal{N}_{i, \neq}}\left(\boldsymbol{f}_{k}^{\varnothing}-\boldsymbol{f}_{i}^{\varnothing}\right) \cdot \int_{\Omega_{i k}} \varphi_{i} \varphi_{k} \hat{\boldsymbol{R}} \mathrm{d} \Omega^{\varnothing}+\boldsymbol{f}_{i}^{\varnothing} \cdot \int_{\Omega_{i k}} \varphi_{i} \hat{\boldsymbol{R}} \mathrm{d} \Omega^{\varnothing} \\
& =\sum_{k \in \mathcal{N}_{i, \neq}}\left(\boldsymbol{f}_{k}^{\varnothing}-\boldsymbol{f}_{i}^{\varnothing}\right) \cdot \int_{\Omega_{i k}} \varphi_{i} \varphi_{k} \hat{\boldsymbol{R}} \mathrm{d} \Omega^{\varnothing}+\boldsymbol{f}_{i}^{\varnothing} \cdot \widehat{\boldsymbol{L}}_{i}^{\varnothing},
\end{aligned}
$$

which can be substituted into (A.8) to give (12).

\section{REFERENCES}

1. Goldstine HH, von Neumann J. Blast wave calculations. Communications on Pure and Applied Mathematics 1955; 8:327-354.

2. Brode HL. Numerical solutions of spherical blast waves. Journal of Applied Physics 1955; 26:766-775.

3. Payne RB. A numerical method for a converging cylindrical shock. Journal of Fluid Mechanics 1957; 2:185-200.

4. Sod GA. A numerical study of a converging cylindrical shock. Journal of Fluid Mechanics 1977; 83(04):785-794. http://www.journals.cambridge.org/abstract_S0022112077001463.

5. Sedov LI. Similarity and dimensional methods in mechanics. Academic Press, 1959.

6. Bagabir A, Drikakis D. Numerical experiments using high-resolution schemes for unsteady, inviscid, compressible flows. Computer Methods in Applied Mechanics and Engineering 2004; 193(42-44):4675-4705. http://linkinghub. elsevier.com/retrieve/pii/S0045782504002312.

7. Liu TG, Khoo BC, Yeo KS. The numerical simulations of explosion and implosion in air: use of a modified Harten's TVD scheme. International Journal for Numerical Methods in Fluids 1999; 31:661-680.

8. Harten A. High resolution schemes for hyperbolic conservation laws. Journal of Computational Physics 1983; 49(1):151-164. http://linkinghub.elsevier.com/retrieve/pii/0021999183901365http://linkinghub.elsevier.com/ retrieve/pii/0021999183901183.

9. Li S. WENO Schemes for Cylindrical and Spherical Geometry. Technical Report 1, Los Alamos National Laboratory LA-UR-03-8922, 2003. http://math.lanl.gov/shenli/publications/weno_sph.pdf.

10. Dutta S, Glimm J, Grove JW, Sharp DH, Zhang Y. Spherical Richtmyer-Meshkov instability for axisymmetric flow. Mathematics and Computers in Simulation 2004; 65(4-5):417-430. http://linkinghub.elsevier.com/retrieve/pii/ S0378475404000266.

11. Maire PH. A high-order cell-centred Lagrangian scheme for compressible fluid flows in two-dimensional cylindrical geometry. Journal of Computational Physics 2009; 228(18):6882-6915. http://linkinghub.elsevier.com/retrieve/pii/ S0021999109003283.

12. Illenseer TF, Duschl WJ. Two-dimensional central-upwind schemes for curvilinear grids and application to gas dynamics with angular momentum. Computer Physics Communications 2009; 180(11):2283-2302. http://linkinghub. elsevier.com/retrieve/pii/S0010465509002367.

13. Clain S, Rochette D, Touzani R. A multislope MUSCL method on unstructured meshes applied to compressible Euler equations for axisymmetric swirling flows. Journal of Computational Physics 2010; 229(13):4884-4906. http://linkinghub.elsevier.com/retrieve/pii/S0021999110001142.

14. Cheng J, Shu CW. A cell-centered Lagrangian scheme with the preservation of symmetry and conservation properties for compressible fluid flows in two-dimensional cylindrical geometry. Journal of Computational Physics 2010; 229(19):7191-7206. http://linkinghub.elsevier.com/retrieve/pii/S0021999110003220.

15. Loubère R, Maire PH, Shashkov M. ReALE: A Reconnection Arbitrary-LagrangianEulerian method in cylindrical geometry. Computer \& Fluids 2011; 46(1):59-69. http://linkinghub.elsevier.com/retrieve/pii/S0045793010002252.

16. Fezoui L, Stoufflet B. A class of implicit upwind schemes for Euler simulations with unstructured meshes. Journal of Computational Physics 1989; 84(1):174-206. http://linkinghub.elsevier.com/retrieve/pii/0021999189901873.

17. Selmin V. The node-centred finite volume approach: bridge between finite differences and finite elements. Computer Methods in Applied Mechanics and Engineering 1993; 102:107-138.

18. Selmin V, Formaggia L. Unified construction of finite element and finite volume discretizations for compressible flows. Computer Methods in Applied Mechanics and Engineering 1996; 39(1):1-32. http://www3.interscience.wiley. com/journal/16352/abstract. 
19. Guardone A, Vigevano L. Finite element/volume solution to axisymmetric conservation laws. Journal of Computational Physics 2007; 224:489-518.

20. Persico G, Rebay S. A penalty formulation for the throughflow modeling of turbomachinery. Computers \& Fluids 2012; 60:86-98. http://linkinghub.elsevier.com/retrieve/pii/S0045793012000886.

21. Guardone A, De Santis D, Geraci G, Pasta M. On the relation between finite element and finite volume schemes for compressible flows with cylindrical and spherical symmetry. Journal of Computational Physics 2011; 230(3):680-694. http://linkinghub.elsevier.com/retrieve/pii/S0021999110005577.

22. Toro EF. Riemann solvers and numerical methods for fluid mechanics. Springer: Berlin, 1997.

23. Quarteroni A. Valli A, Numerical approximation of partial differential equations. No. 23 in Springer series in computational mathematics, Springer-Verlag, 1994.

24. Donea J, Huerta A. Finite element methods for flow problems. Wiley, 2002.

25. Ern A, Guermond JL. Theory and Practice of Finite Elements, Applied Mathematical Science, Vol. 159. SpringerVerlag: New York, 2004.

26. LeVeque RJ. Finite volume methods for conservation laws and hyperbolic systems. Cambridge University Press, 2002.

27. Roe PL. Approximate Riemann solvers, parameter vectors, and difference schemes. Journal of Computational Physics 1981; 43(2):357-372. http://linkinghub.elsevier.com/retrieve/pii/0021999181901285.

28. Guardone A, Vigevano L. Roe linearization for the van der Waals gas. Journal of Computational Physics 2002; 175:50-78.

29. van Leer B. Towards the ultimate conservative difference scheme II. Monotoniticy and conservation combined in a second order scheme. Journal of Computational Physics 1974; 14:361-370.

30. Jameson A. Time dependent calculations using multigrid, with applications to unsteady flows past airfoils and wings. 10th AIAA Computational Fluid Dynamics Conference, Honolulu, HI, 1991; 1-13. http://aero-comlab.stanford.edu/ Papers/jameson.aiaa.91-1596.pdf.

31. Saad Y. SPARSEKIT: A Basic Toll Kit for Sparse Matrix Computations. Technical Report, University of Minnesota, Computer Science Department, 1994.

32. Rebay S. Efficient unstructured mesh generation by means of Delaunay triangulation and Bowyer-Watson algorithm. Journal of Computational Physics 1993; 106:125-138.

33. Guderley G. Powerful spherical and cylindrical compression shocks in the neighbourhood of the centre and of the cylinder axis. Luftfahrtforschung 1942; 19:302-312.

34. Ponchaut NF, Hornung HG, Pullin DI, Mouton CA. On imploding cylindrical and spherical shock waves in a perfect gas. Journal of Fluid Mechanics 2006; 560:103. http://www.journals.cambridge.org/abstract_S0022112006000590.

35. Rider WJ. Revisiting Wall Heating. Journal of Computational Physics 2000; 162(2):395-410. http://linkinghub. elsevier.com/retrieve/pii/S0021999100965446.

36. Takayama K, Kleine H, Grönig H. An experimental investigation of the stability of converging cylindrical shock waves in air. Experiments in Fluids 1987; 5(5):315-322. http://www.springerlink.com/index/N815246300V87606. pdf.

37. Watanabe M, Takayama K. Stability of converging cylindrical shock waves. Shock Waves 1991; 1(2):149-160. http://www.springerlink.com/index/X19318LK65T261NJ.pdf.

38. Breil J, Galera S, Maire PH. Multi-material ALE computation in inertial confinement fusion code CHIC. Computer \& Fluids 2011; 46(1):161-167. http://linkinghub.elsevier.com/retrieve/pii/S0045793010001489.

39. Eliasson V, Apazidis N, Tillmark N, Lesser MB. Focusing of Strong Shocks in an Annular Shock Tube. Shock Waves 2006; 15(3-4):205-217. http://www.springerlink.com/index/10.1007/s00193-006-0035-0.

40. Eliasson V, Apazidis N, Tillmark N. Controlling the form of strong converging shocks by means of disturbances. Shock Waves 2007; 17:29-42.

41. Eliasson V, Henshaw WD, Appelö D. On cylindrically converging shock waves shaped by obstacles. Physica D: Nonlinear Phenomena 2008; 237(14-17):2203-2209. http://linkinghub.elsevier.com/retrieve/pii/S0167278907004186.

42. Shi H, Yamamura K. The interaction between shock waves and solid spheres arrays in a shock tube. Acta Mechanica Sinica 2004; 20(3):219-227. http://www.cqvip.com/qk/86601x/2004003/10.313104.html. 